\title{
AN APPLICATION OF COLLAPSING LEVELS TO THE REPRESENTATION THEORY OF AFFINE VERTEX ALGEBRAS
}

\author{
DRAŽEN ADAMOVIĆ, VICTOR G. KAC, PIERLUIGI MÖSENEDER FRAJRIA, PAOLO PAPI, \\ AND OZREN PERŠE
}

\begin{abstract}
We discover a large class of simple affine vertex algebras $V_{k}(\mathfrak{g})$, associated to basic Lie superalgebras $\mathfrak{g}$ at non-admissible collapsing levels $k$, having exactly one irreducible $\mathfrak{g}$-locally finite module in the category $\mathcal{O}$. In the case when $\mathfrak{g}$ is a Lie algebra, we prove a complete reducibility result for $V_{k}(\mathfrak{g})$-modules at an arbitrary collapsing level. We also determine the generators of the maximal ideal in the universal affine vertex algebra $V^{k}(\mathfrak{g})$ at certain negative integer levels. Considering some conformal embeddings in the simple affine vertex algebras $V_{-1 / 2}\left(C_{n}\right)$ and $V_{-4}\left(E_{7}\right)$, we surprisingly obtain the realization of non-simple affine vertex algebras of types $B$ and $D$ having exactly one non-trivial ideal.
\end{abstract}

\section{INTRODUCTION}

Affine vertex algebras are one of the most interesting and important classes of vertex algebras. Categories of modules for simple affine vertex algebra $V_{k}(\mathfrak{g})$, associated to a simple Lie algebra $\mathfrak{g}$, have mostly been studied in the case of positive integer levels $k \in \mathbb{Z}_{\geq 0}$. These categories enjoy many nice properties such as: finitely many irreducibles, semisimplicity, modular invariance of characters (cf. 26, 31, 33, 41]).

In recent years, affine vertex algebras have attracted a lot of attention because of their connection with affine $\mathcal{W}$-algebras $W_{k}(\mathfrak{g}, f)$, obtained by quantum Hamiltonian reduction (cf. 221, 23], 34, [35]). Since the quantum Hamiltonian reduction functor $H_{f}(\cdot)$ maps any integrable $\widehat{\mathfrak{g}}$-module to zero (cf. [12, 34]), in order to obtain interesting $\mathcal{W}$-algebras, one has to consider affine vertex algebras $V_{k}(\mathfrak{g})$, for $k \notin \mathbb{Z}_{\geq 0}$.

It turns out that for certain non-admissible levels $k$ (such as negative integer levels), the associated vertex algebras $V_{k}(\mathfrak{g})$ have finitely many irreducibles in category $\mathcal{O}$ (cf. 15, 17, 40), and their characters satisfy certain modular-like properties (cf. [14]). These affine vertex algebras then give $C_{2}$-cofinite $\mathcal{W}$-algebras $W_{k}(\mathfrak{g}, f)$, for properly chosen nilpotent element $f$ (cf. [36, 38]).

In this paper, we classify irreducible modules in the category $K L_{k}$ (i.e. the category of $\mathfrak{g}$-locally finite $V_{k}(\mathfrak{g})$-modules in $\mathcal{O}^{k}$ (see Subsection[2.3) ) for a large family of collapsing levels $k$. Recall from 4 that a level $k$ is called collapsing if the simple $\mathcal{W}$-algebra $W_{k}(\mathfrak{g}, \theta)$, associated to a minimal nilpotent element $e_{-\theta}$, is isomorphic to its affine vertex subalgebra $\mathcal{V}_{k}\left(\mathfrak{g}^{\natural}\right)$ (see Definition 2.2 and (2.7)). In the present paper we keep the notation of 4. In particular, the highest root is normalized by the condition $(\theta, \theta)=2$. We discover a large family of vertex algebras having one irreducible module in the category $K L_{k}$, which in a way extends the results on Deligne series from [15]. Part (1) is proven there in the Lie algebra case.

Theorem 1.1. Assume that the level $k$ and the basic simple Lie superalgebra $\mathfrak{g}$ satisfy one of the following conditions:

(1) $k=-\frac{h^{\vee}}{6}-1$ and $\mathfrak{g}$ is one of the Lie algebras of exceptional Deligne's series $A_{2}, G_{2}, D_{4}, F_{4}$, $E_{6}, E_{7}, E_{8}$, or $\mathfrak{g}=\operatorname{psl}(m \mid m)(m \geq 2), \operatorname{osp}(n+8 \mid n)(n \geq 2), \operatorname{spo}(2 \mid 1), F(4), G(3)$ (for both choices of $\theta$ );

(2) $k=-h^{\vee} / 2+1$ and $\mathfrak{g}=o s p(n+4 m+8 \mid n), n \geq 2, m \geq 0$.

(3) $k=-h^{\vee} / 2+1$ and $\mathfrak{g}=D_{2 m}, m \geq 2$.

(4) $k=-10$ and $\mathfrak{g}=E_{8}$. 
Then $V_{k}(\mathfrak{g})$ is the unique irreducible $V_{k}(\mathfrak{g})$-module in the category $K L_{k}$.

We also prove a complete reducibility result in $K L_{k}$ (cf. Theorem [5.9, Theorem [5.7):

Theorem 1.2. Assume that $\mathfrak{g}$ is a Lie algebra and $k \in \mathbb{C} \backslash \mathbb{Z}_{\geq 0}$. Then $K L_{k}$ is a semi-simple category in the following cases:

- $k$ is a collapsing level.

- $W_{k}(\mathfrak{g}, \theta)$ is a rational vertex operator algebra.

It is interesting that in some cases we have that $K L_{k}$ is a semi-simple category, but there can exist indecomposable but not irreducible $V_{k}(\mathfrak{g})$-modules in the category $\mathcal{O}$. In order to prove Theorem 1.2 we modified methods from 28 and 20] in a vertex algebraic setting. In particular we prove that the contravariant functor $M \mapsto M^{\sigma}$ from [20] acts on the category $K L_{k}$ (cf. Lemma 3.6). Then for the proof of complete reducibility in $K L_{k}$ it is enough to check that every highest weight $V_{k}(\mathfrak{g})$-module in $K L_{k}$ is irreducible (cf. Theorem 5.5).

Representation theory of a simple affine vertex algebra $V_{k}(\mathfrak{g})$ is naturally connected with the structure of the maximal ideal in the universal affine vertex algebra $V^{k}(\mathfrak{g})$. In the second part of paper we present explicit formulas for singular vectors which generate the maximal ideal in $V^{2-2 \ell}\left(D_{2 \ell}\right)$ (which is case (3) of Theorem 1.1) and $V^{-2}\left(D_{\ell}\right)$. In the second case, we show that the Hamiltonian reduction functor $H_{\theta}(\cdot)$ gives an equivalence of the category of $\mathfrak{g}$-locally finite $V_{-2}\left(D_{\ell}\right)$-modules $K L_{-2}$ and the category of modules for a rational vertex algebra $V_{\ell-4}\left(A_{1}\right)$. Singular vectors in $V^{k}(\mathfrak{g})$ for certain negative integer levels $k$ have also been constructed in [2].

We also apply our results to study the structure of conformally embedded subalgebras of some simple affine vertex algebras.

As in [6], for a subalgebra $\mathfrak{k}$ of a simple Lie algebra $\mathfrak{g}$, we denote by $\widetilde{V}(k, \mathfrak{k})$ the vertex subalgebra of $V_{k}(\mathfrak{g})$ generated by $x(-1) \mathbf{1}, x \in \mathfrak{k}$. If $\mathfrak{k}$ is a reductive quadratic subalgebra of $\mathfrak{g}$, then we say that $\widetilde{V}(k, \mathfrak{k})$ is conformally embedded in $V_{k}(\mathfrak{g})$ if the Sugawara-Virasoro vectors of both algebras coincide. We also say that $\mathfrak{k}$ is conformally embedded in $\mathfrak{g}$ at level $k$ if $\widetilde{V}(k, \mathfrak{k})$ is conformally embedded in $V_{k}(\mathfrak{g})$.

We are able to prove that in the cases listed in Theorem 1.3 below, $\widetilde{V}(k, \mathfrak{k})$ is not simple. On the other hand, we show that $V_{-1 / 2}\left(C_{5}\right)$ contains a simple subalgebra $V_{-2}\left(B_{2}\right) \otimes V_{-5 / 2}\left(A_{1}\right)$ (see Corollary (7.4). For the conformal embedding of $D_{6} \times A_{1}$ into $E_{7}$ at level $k=-4$, we show that $\widetilde{V}\left(-4, D_{6} \times A_{1}\right)=\mathcal{V}_{-4}\left(D_{6}\right) \otimes V_{-4}\left(A_{1}\right)$ where $\mathcal{V}_{-4}\left(D_{6}\right)$ is a quotient of the universal affine vertex algebra $V^{-4}\left(D_{6}\right)$ by two singular vectors of conformal weights two and three (cf. (9.6)). Moreover, $\mathcal{V}_{-4}\left(D_{6}\right)$ has infinitely many irreducible modules in the category of $\mathfrak{g}$-locally finite modules, which we explicitly describe. All of them appear in $V_{-4}\left(E_{7}\right)$ as submodules or subquotients.

Theorem 1.3. Let $\mathcal{V}_{k}\left(D_{\ell}\right), \mathcal{V}_{k}\left(B_{\ell}\right)$, be the vertex algebras defined in (6.3), (7.1), (9.6). Consider the following conformal embeddings:

(1) $D_{\ell} \times A_{1}$ into $C_{2 l}$ for $\ell \geq 4$ at level $k=-\frac{1}{2}$.

(2) $B_{\ell} \times A_{1}$ into $C_{2 l+1}$ for $\ell \geq 3$ at level $k=-\frac{1}{2}$.

(3) $D_{6} \times A_{1}$ into $E_{7}$ at level $k=-4$.

Then,

- $\widetilde{V}\left(-\frac{1}{2}, D_{\ell} \times A_{1}\right)=\mathcal{V}_{-2}\left(D_{\ell}\right) \otimes V_{-\ell}\left(A_{1}\right)$ in case (1),

- $\widetilde{V}\left(-\frac{1}{2}, B_{\ell} \times A_{1}\right)=\mathcal{V}_{-2}\left(B_{\ell}\right) \otimes V_{-\ell-1 / 2}\left(A_{1}\right)$ in case (2),

- $\widetilde{V}\left(-4, D_{6} \times A_{1}\right)=\mathcal{V}_{-4}\left(D_{6}\right) \otimes V_{-4}\left(A_{1}\right)$ in case (3).

Moreover, the algebras $\mathcal{V}_{k}\left(D_{\ell}\right), \mathcal{V}_{k}\left(B_{\ell}\right)$, are non-simple, with a unique non-trivial ideal.

The decompositions of the embeddings above is still an open problem, and will be a subject of our forthcoming papers.

Acknowledgement. We would like to thank Maria Gorelik, Tomoyuki Arakawa and Anne Moreau for correspondence and discussions. 


\section{Preliminaries}

We assume that the reader is familiar with the notion of vertex (super)algebra (cf. [18, 25, 32]) and of simple basic Lie superalgebras (see 30]) and their affinizations (see 31 for the Lie algebra case).

Let $V$ be a conformal vertex algebra. Denote by $A(V)$ the associative algebra introduced in [41, called the Zhu algebra of $V$.

2.1. Basic Lie superalgebras and minimal gradings. For the reader's convenience we recall here the setting and notation of 4 regarding basic Lie superalgebras and their minimal gradings. Let $\mathfrak{g}=\mathfrak{g}_{\overline{0}} \oplus \mathfrak{g}_{\overline{1}}$ be a simple finite dimensional basic Lie superalgebra. We choose a Cartan subalgebra $\mathfrak{h}$ for $\mathfrak{g}_{\overline{0}}$ and let $\Delta$ be the set of roots. Assume $\mathfrak{g}$ is not $\operatorname{osp}(3 \mid n)$. A root $-\theta$ is called minimal if it is even and there exists an additive function $\varphi: \Delta \rightarrow \mathbb{R}$ such that $\varphi_{\mid \Delta} \neq 0$ and $\varphi(\theta)>\varphi(\eta), \forall \eta \in \Delta \backslash\{\theta\}$. Fix a minimal root $-\theta$ of $\mathfrak{g}$. We may choose root vectors $e_{\theta}$ and $e_{-\theta}$ such that

$$
\left[e_{\theta}, e_{-\theta}\right]=x \in \mathfrak{h}, \quad\left[x, e_{ \pm \theta}\right]= \pm e_{ \pm \theta} .
$$

Due to the minimality of $-\theta$, the eigenspace decomposition of ad $x$ defines a minimal $\frac{1}{2} \mathbb{Z}$-grading ([35, $(5.1)])$ :

$$
\mathfrak{g}=\mathfrak{g}_{-1} \oplus \mathfrak{g}_{-1 / 2} \oplus \mathfrak{g}_{0} \oplus \mathfrak{g}_{1 / 2} \oplus \mathfrak{g}_{1},
$$

where $\mathfrak{g}_{ \pm 1}=\mathbb{C} e_{ \pm \theta}$. We thus have a bijective correspondence between minimal gradings (up to an automorphism of $\mathfrak{g}$ ) and minimal roots (up to the action of the Weyl group). Furthermore, one has

$$
\mathfrak{g}_{0}=\mathfrak{g}^{\natural} \oplus \mathbb{C} x, \quad \mathfrak{g}^{\natural}=\left\{a \in \mathfrak{g}_{0} \mid(a \mid x)=0\right\} .
$$

Note that $\mathfrak{g}^{\natural}$ is the centralizer of the triple $\left\{f_{\theta}, x, e_{\theta}\right\}$. We can choose $\mathfrak{h}^{\natural}=\{h \in \mathfrak{h} \mid(h \mid x)=0\}$, as a Cartan subalgebra of the Lie superalgebra $\mathfrak{g}^{\natural}$, so that $\mathfrak{h}=\mathfrak{h}^{\natural} \oplus \mathbb{C} x$.

For a given choice of a minimal root $-\theta$, we normalize the invariant bilinear form $(\cdot \mid \cdot)$ on $\mathfrak{g}$ by the condition

$$
(\theta \mid \theta)=2 .
$$

The dual Coxeter number $h^{\vee}$ of the pair $(\mathfrak{g}, \theta)$ (equivalently, of the minimal gradation (2.1)) is defined to be half the eigenvalue of the Casimir operator of $\mathfrak{g}$ corresponding to $(\cdot \mid \cdot)$, normalized by (2.3). Since $\theta$ is the highest root, we have that $2 h^{\vee}=(\theta \mid \theta+2 \rho)$ hence

$$
(\rho \mid \theta)=h^{\vee}-1 \text {. }
$$

The complete list of the Lie superalgebras $\mathfrak{g}^{\natural}$, the $\mathfrak{g}^{\natural}$-modules $\mathfrak{g}_{ \pm 1 / 2}$ (they are isomorphic and selfdual), and $h^{\vee}$ for all possible choices of $\mathfrak{g}$ and of $\theta$ (up to isomorphism) is given in Tables 1,2,3 of 35. We reproduce them below. Note that in these tables $\mathfrak{g}=\operatorname{osp}(m \mid n)($ resp. $\mathfrak{g}=\operatorname{spo}(n \mid m))$ means that $\theta$ is the highest root of the simple component $s o(m)$ (resp. $s p(n))$ of $\mathfrak{g}_{\overline{0}}$. Also, for $\mathfrak{g}=s l(m \mid n)$ or $\operatorname{psl}(m \mid m)$ we always take $\theta$ to be the highest root of the simple component $\operatorname{sl}(m)$ of $\mathfrak{g}_{\overline{0}}$ (for $m=4$ we take one of the simple roots). Note that the exceptional Lie superalgebras $\mathfrak{g}=F(4)$ and $\mathfrak{g}=G(3)$ appear in both Tables 2 and 3, which corresponds to the two inequivalent choices of $\theta$, the first one being a root of the simple component $\operatorname{sl}(2)$ of $\mathfrak{g}_{\overline{0}}$.

Table 1

$\mathfrak{g}$ is a simple Lie algebra.

\begin{tabular}{c|c|c|c||c|c|c|c}
$\mathfrak{g}$ & $\mathfrak{g}^{\natural}$ & $\mathfrak{g}_{1 / 2}$ & $h^{\vee}$ & $\mathfrak{g}$ & $\mathfrak{g}^{\natural}$ & $\mathfrak{g}_{1 / 2}$ & $h^{\vee}$ \\
\hline$s l(n), n \geq 3$ & $g l(n-2)$ & $\mathbb{C}^{n-2} \oplus\left(\mathbb{C}^{n-2}\right)^{*}$ & $n$ & $F_{4}$ & $s p(6)$ & $\bigwedge_{0}^{3} \mathbb{C}^{6}$ & 9 \\
\hline$s o(n), n \geq 5$ & $s l(2) \oplus \operatorname{so}(n-4)$ & $\mathbb{C}^{2} \otimes \mathbb{C}^{n-4}$ & $n-2$ & $E_{6}$ & $\operatorname{sl}(6)$ & $\bigwedge^{3} \mathbb{C}^{6}$ & 12 \\
\hline$s p(n), n \geq 2$ & $s p(n-2)$ & $\mathbb{C}^{n-2}$ & $n / 2+1$ & $E_{7}$ & $\operatorname{so}(12)$ & $\operatorname{spin}_{12}$ & 18 \\
\hline$G_{2}$ & $s l(2)$ & $S^{3} \mathbb{C}^{2}$ & 4 & $E_{8}$ & $E_{7}$ & $\operatorname{dim}=56$ & 30
\end{tabular}




\begin{tabular}{l|c|c|c||c|c|c|c}
$\mathfrak{g}$ & $\mathfrak{g}^{\natural}$ & $\mathfrak{g}_{1 / 2}$ & $h^{\vee}$ & $\mathfrak{g}$ & $\mathfrak{g}^{\natural}$ & $\mathfrak{g}_{1 / 2}$ & $h^{\vee}$ \\
\hline $\begin{array}{l}s l(2 \mid m), \\
m \neq 2\end{array}$ & $g l(m)$ & $\mathbb{C}^{m} \oplus\left(\mathbb{C}^{m}\right)^{*}$ & $2-m$ & $D(2,1 ; a)$ & $\operatorname{sl}(2) \oplus \operatorname{sl}(2)$ & $\mathbb{C}^{2} \otimes \mathbb{C}^{2}$ & 0 \\
\hline$p s l(2 \mid 2)$ & $s l(2)$ & $\mathbb{C}^{2} \oplus \mathbb{C}^{2}$ & 0 & $F(4)$ & $\operatorname{so}(7)$ & $\operatorname{spin} 7$ & -2 \\
\hline $\operatorname{spo}(2 \mid m)$ & $s o(m)$ & $\mathbb{C}^{m}$ & $2-m / 2$ & $G(3)$ & $G_{2}$ & $\operatorname{Dim}=0 \mid 7$ & $-3 / 2$ \\
\hline$o s p(4 \mid m)$ & $s l(2) \oplus \operatorname{sp}(m)$ & $\mathbb{C}^{2} \otimes \mathbb{C}^{m}$ & $2-m$ & &
\end{tabular}

Table 3

Both $\mathfrak{g}$ and $\mathfrak{g}^{\natural}$ are not Lie algebras $(m, n \geq 1)$.

\begin{tabular}{c|c|c|c}
$\mathfrak{g}$ & $\mathfrak{g}^{\natural}$ & $\mathfrak{g}_{1 / 2}$ & $h^{\vee}$ \\
\hline$s l(m \mid n), m \neq n, m>2$ & $g l(m-2 \mid n)$ & $\mathbb{C}^{m-2 \mid n} \oplus\left(\mathbb{C}^{m-2 \mid n}\right)^{*}$ & $m-n$ \\
\hline$p s l(m \mid m), m>2$ & $s l(m-2 \mid m)$ & $\mathbb{C}^{m-2 \mid m} \oplus\left(\mathbb{C}^{m-2 \mid m}\right)^{*}$ & 0 \\
\hline$s p o(n \mid m), n \geq 4$ & $s p o(n-2 \mid m)$ & $\mathbb{C}^{n-2 \mid m}$ & $1 / 2(n-m)+1$ \\
\hline$o s p(m \mid n), m \geq 5$ & $o s p(m-4 \mid n) \oplus \operatorname{sl}(2)$ & $\mathbb{C}^{m-4 \mid n} \otimes \mathbb{C}^{2}$ & $m-n-2$ \\
\hline$F(4)$ & $D(2,1 ; 2)$ & $\operatorname{Dim}=6 \mid 4$ & 3 \\
\hline$G(3)$ & $o s p(3 \mid 2)$ & $\operatorname{Dim}=4 \mid 4$ & 2
\end{tabular}

In this paper we shall exclude the case of $\mathfrak{g}=\operatorname{sl}(n+2 \mid n), n>0$. In all other cases the Lie superalgebra $\mathfrak{g}^{\natural}$ decomposes in a direct sum of all its minimal ideals, called components of $\mathfrak{g}^{\natural}$ :

$$
\mathfrak{g}^{\natural}=\bigoplus_{i \in I} \mathfrak{g}_{i}^{\natural},
$$

where each summand is either the (at most 1-dimensional) center of $\mathfrak{g}^{\natural}$ or is a basic simple Lie superalgebra different from $\operatorname{psl}(n \mid n)$. Let $C_{\mathfrak{g}_{i}^{\natural}}$ be the Casimir operator of $\mathfrak{g}_{i}^{\natural}$ corresponding to $(\cdot \mid \cdot)_{\mid \mathfrak{g}_{i}^{\natural} \times \mathfrak{g}_{i}^{\natural}}$. We define the dual Coxeter number $h_{0, i}^{\vee}$ of $\mathfrak{g}_{i}^{\natural}$ as half of the eigenvalue of $C_{\mathfrak{g}_{i}^{\natural}}$ acting on $\mathfrak{g}_{i}^{\natural}$ (which is 0 if $\mathfrak{g}_{i}^{\natural}$ is abelian).

Denote by $V_{\mathfrak{g}}(\mu)$ (or $V(\mu)$ ) the irreducible finite-dimensional highest weight $\mathfrak{g}$-module with highest weight $\mu$. Denote by $P_{+}$the set of highest weights of irreducible finite-dimensional representations of $\mathfrak{g}$.

Since $\mathfrak{h}=\mathfrak{h}^{\natural} \oplus \mathbb{C} x$, we have, in particular, that $\mu \in \mathfrak{h}^{*}$ can be uniquely written as

$$
\mu=\mu_{\mid \mathfrak{h}^{\natural}}+\ell \theta,
$$

with $\ell \in \mathbb{C}$. If $\mu \in P_{+}$, then, since $\theta\left(\mathfrak{h}^{\natural}\right)=0, \mu\left(\theta^{\vee}\right)=2 \ell \in \mathbb{Z}$, so $\ell \in \frac{1}{2} \mathbb{Z}_{\geq 0}$.

2.2. Affine Lie algebras, vertex algebras, $\mathcal{W}$-algebras. Let $\widehat{\mathfrak{g}}$ be the affinization of $\mathfrak{g}$ :

$$
\widehat{\mathfrak{g}}=\mathbb{C}\left[t, t^{-1}\right] \otimes \mathfrak{g} \oplus \mathbb{C} K \oplus \mathbb{C} d
$$

with the usual commutation relations. We let $\delta$ be the fundamental imaginary root. Let $\alpha_{0}=\delta-\theta$ the affine simple root. Since $\theta$ is even, hence non-isotropic, so that $\alpha_{0}^{\vee}=K-\theta^{\vee}$ makes sense.

Denote by $L(\lambda)$ (or $L_{\mathfrak{g}}(\lambda)$ ) the irreducible highest weight $\widehat{\mathfrak{g}}-$ module with highest weight $\lambda$.

Denote by $V^{k}(\mathfrak{g})$ the universal affine vertex algebra associated to $\widehat{\mathfrak{g}}$ of level $k \in \mathbb{C}$. We shall assume that $k \neq-h^{\vee}$. Then (see e.g. 32) $V^{k}(\mathfrak{g})$ is a conformal vertex algebra with Segal-Sugawara conformal vector $\omega_{\mathfrak{g}}$. Let $Y\left(\omega_{\mathfrak{g}}, z\right)=\sum L_{\mathfrak{g}}(n) z^{-n-2}$ be the corresponding Virasoro field. Denote by $V_{k}(\mathfrak{g})$ the (unique) simple quotient of $V^{k}(\mathfrak{g})$. Clearly, $V_{k}(\mathfrak{g}) \cong L_{\mathfrak{g}}\left(k \Lambda_{0}\right)$ as $\widehat{\mathfrak{g}}-$ modules.

Denote by $W^{k}(\mathfrak{g}, \theta)$ the affine $\mathcal{W}$-algebra obtained from $V^{k}(\mathfrak{g})$ by Hamiltonian reduction relative to a minimal nilpotent element $e_{-\theta}$. Denote by $W_{k}(\mathfrak{g}, \theta)$ the simple quotient of $W^{k}(\mathfrak{g}, \theta)$. Recall that the vertex algebra $W^{k}(\mathfrak{g}, \theta)$ is strongly and freely generated by elements $J^{\{a\}}$, where $a$ runs over a basis of $\mathfrak{g}^{\natural}, G^{\{v\}}$, where $v$ runs over a basis of $\mathfrak{g}_{-1 / 2}$, and the Virasoro vector $\omega$. The elements $J^{\{a\}}, G^{\{v\}}$ are primary of conformal weight 1 and $3 / 2$, respectively, with respect to $\omega$.

Let $\mathcal{V}^{k}\left(\mathfrak{g}^{\natural}\right)$ be the subalgebra of the vertex algebra $W^{k}(\mathfrak{g}, \theta)$, generated by $\left\{J^{\{a\}} \mid a \in \mathfrak{g}^{\natural}\right\}$. The vertex algebra $\mathcal{V}^{k}\left(\mathfrak{g}^{\natural}\right)$ is isomorphic to a universal affine vertex algebra. More precisely, letting

$$
k_{i}=k+\frac{1}{2}\left(h^{\vee}-h_{0, i}^{\vee}\right), i \in I,
$$

the map $a \mapsto J^{\{a\}}$ extends to an isomorphism $\mathcal{V}^{k}\left(\mathfrak{g}^{\natural}\right) \simeq \bigotimes_{i \in I} V^{k_{i}}\left(\mathfrak{g}_{i}^{\natural}\right)$. 
We also set $\mathcal{V}_{k}\left(\mathfrak{g}^{\natural}\right)$ to be the image of $\mathcal{V}^{k}\left(\mathfrak{g}^{\natural}\right)$ in $W_{k}(\mathfrak{g}, \theta)$. Clearly we can write

$$
\mathcal{V}_{k}\left(\mathfrak{g}^{\natural}\right) \simeq \bigotimes_{i \in I} \mathcal{V}_{k_{i}}\left(\mathfrak{g}_{i}^{\natural}\right)
$$

where $\mathcal{V}_{k_{i}}\left(\mathfrak{g}_{i}^{\natural}\right)$ is some quotient (not necessarily simple) of $V^{k_{i}}\left(\mathfrak{g}_{i}^{\natural}\right)$.

2.3. Category $\mathcal{O}$ and Hamiltonian reduction functor. Recall that $\widehat{\mathfrak{g}}$-module $M$ is in category $\mathcal{O}^{k}$ if it is $\widehat{\mathfrak{h}}$-diagonalizable with finite dimensional weight spaces, $K$ acts as $k I d_{M}$ and $M$ has a finite number of maximal weights.

There is a remarkable functor $H_{\theta}$ from $\mathcal{O}^{k}$ to the category of $W^{k}(\mathfrak{g}, \theta)$-modules whose properties will be very important in the following. We recall them in a form suitable for our purposes (see [12] for details; there $H_{\theta}$ is denoted by $H^{0}$ ).

\section{Theorem 2.1.}

(1) $H_{\theta}$ is exact.

(2) If $L(\lambda)$ is a irreducible highest weight $\widehat{\mathfrak{g}}$-module, then $\lambda\left(\alpha_{0}^{\vee}\right) \in \mathbb{Z}_{\geq 0}$ implies $H_{\theta}(L(\lambda))=\{0\}$. Otherwise $H_{\theta}(L(\lambda))$ is isomorphic to the irreducible $W^{k}(\mathfrak{g}, \theta)$-module with highest weight $\phi_{\lambda}$ defined by formula (67) in 12 .

\subsection{Collapsing levels.}

Definition 2.2. Assume $k \neq-h^{\vee}$. If $W_{k}(\mathfrak{g}, \theta)=\mathcal{V}_{k}\left(\mathfrak{g}^{\natural}\right)$, we say that $k$ is a collapsing level.

Theorem 2.3. [4, Theorem 3.3] Let $p(k)$ be the polynomial listed in Table 4 below. Then $k$ is a collapsing level if and only if $k \neq-h^{\vee}$ and $p(k)=0$. In such cases,

$$
W_{k}(\mathfrak{g}, \theta)=\bigotimes_{i \in I^{*}} V_{k_{i}}\left(\mathfrak{g}_{i}^{\natural}\right)
$$

where $I^{*}=\left\{i \in I \mid k_{i} \neq 0\right\}$. If $I^{*}=\emptyset$, then $W_{k}(\mathfrak{g}, \theta)=\mathbb{C}$.

Table 4

Polynomials $p(k)$.

\begin{tabular}{c|c||c|c}
$\mathfrak{g}$ & $p(k)$ & $\mathfrak{g}$ & $p(k)$ \\
\hline$s l(m \mid n), n \neq m$ & $(k+1)(k+(m-n) / 2)$ & $E_{6}$ & $(k+3)(k+4)$ \\
\hline$p s l(m \mid m)$ & $k(k+1)$ & $E_{7}$ & $(k+4)(k+6)$ \\
\hline$o s p(m \mid n)$ & $(k+2)(k+(m-n-4) / 2)$ & $E_{8}$ & $(k+6)(k+10)$ \\
\hline$s p o(n \mid m)$ & $(k+1 / 2)(k+(n-m+4) / 4)$ & $F_{4}$ & $(k+5 / 2)(k+3)$ \\
\hline$D(2,1 ; a)$ & $(k-a)(k+1+a)$ & $G_{2}$ & $(k+4 / 3)(k+5 / 3)$ \\
\hline$F(4), \mathfrak{g}^{\natural}=s o(7)$ & $(k+2 / 3)(k-2 / 3)$ & $G(3), \mathfrak{g}^{\natural}=G_{2}$ & $(k-1 / 2)(k+3 / 4)$ \\
\hline$F(4), \mathfrak{g}^{\natural}=D(2,1 ; 2)$ & $(k+3 / 2)(k+1)$ & $G(3), \mathfrak{g}^{\natural}=o s p(3 \mid 2)$ & $(k+2 / 3)(k+4 / 3)$
\end{tabular}

2.5. Weyl vertex algebra. Let $M_{\ell}$ denote the Weyl vertex algebra (also called symplectic bosons) generated by even elements $a_{i}^{ \pm}, i=1, \ldots, \ell$ satisfying the following $\lambda$-brackets

$$
\left[\left(a_{i}^{ \pm}\right)_{\lambda}\left(a_{j}^{ \pm}\right)\right]=0, \quad\left[\left(a_{i}^{+}\right)_{\lambda}\left(a_{j}^{-}\right)\right]=\delta_{i, j} .
$$

Recall also that the symplectic affine vertex algebra $V_{-1 / 2}\left(C_{\ell}\right)$ is realized as a $\mathbb{Z}_{2}-$ orbifold of $M_{\ell}$ (see 22 ).

\section{The CATEGORY $K L_{k}$}

Let $k$ be a noncritical level. Note that the Casimir element of $\widehat{\mathfrak{g}}$ can be expressed as $\Omega=d+L_{\mathfrak{g}}(0)$; it commutes with $\widehat{\mathfrak{g}}$-action.

Consider the category $\mathcal{C}^{k}$ of modules for the universal affine vertex algebra $V^{k}(\mathfrak{g})$, i.e. the category of restricted $\widehat{\mathfrak{g}}$-modules of level $k$. Regard $M \in \mathcal{C}^{k}$ as a $\widehat{\mathfrak{g}}$-module by letting $d$ act as $-L_{\mathfrak{g}}(0)$. Let $K L^{k}$ be the category of modules $M \in \mathcal{C}^{k}$ such that, as $\widehat{\mathfrak{g}}$-modules, are in $\mathcal{O}^{k}$ and which admit the following weight space decomposition with respect to $L_{\mathfrak{g}}(0)$ :

$$
M=\bigoplus_{\alpha \in \mathbb{C}} M(\alpha), \quad L_{\mathfrak{g}}(0) \mid M(\alpha) \equiv \alpha \mathrm{Id}, \operatorname{dim} M(\alpha)<\infty .
$$


Our definition is related but different from the one introduced in [13. Let $K L_{k}$ be the category of all modules in $K L^{k}$ which are $V_{k}(\mathfrak{g})$-modules.

Remark 3.1. If $V_{k}(\mathfrak{g})$ has finitely many irreducible modules in the category $K L^{k}$, one can show that every $V_{k}(\mathfrak{g})$-module $M$ in $K L_{k}$ is of finite length. This happens when $k$ is admissible (cf. [12]) and when $V_{k}(\mathfrak{g})$ is quasi-lisse (cf. [14]). But when $V_{k}(\mathfrak{g})$ has infinitely many irreducible modules in $K L^{k}$ (as in the cases considered in [39], 11]), then one can have modules in $K L_{k}$ of infinite length.

Recall that there is a one-to-one correspondence between irreducible $\mathbb{Z}_{\geq 0}$-graded modules for a conformal vertex algebra $V$ (with a conformal vector $\omega$, such that $Y(\omega, z)=\sum_{i \in \mathbb{Z}} L(i) z^{-i-2}$ ) and irreducible modules for the corresponding Zhu algebra $A(V)$ [41. This implies, in particular, that there is a one-to-one correspondence between irreducible finite-dimensional $A(V)$-modules and irreducible $\mathbb{Z}_{\geq 0}$-graded $V$-modules whose graded components, which are eigenspaces for $L(0)$, are finite-dimensional. In the case of affine vertex algebras, we have the following simple interpretation.

Proposition 3.2. Let $\widetilde{V}_{k}(\mathfrak{g})$ be a quotient of $V^{k}(\mathfrak{g})$ (not necessary simple). Consider $\widetilde{V}_{k}(\mathfrak{g})$ as a conformal vertex algebra with conformal vector $\omega_{\mathfrak{g}}$. Then there is a one-to-one correspondence between irreducible $\widetilde{V}_{k}(\mathfrak{g})$ in the category $K L^{k}$ and irreducible finite-dimensional $A\left(\widetilde{V}_{k}(\mathfrak{g})\right)$-modules.

Corollary 3.3. Assume that $\mathfrak{g}$ is a simple basic Lie superalgebra and $\widetilde{V}_{k}(\mathfrak{g})$ is a quotient of $V^{k}(\mathfrak{g})$ such that the trivial module $\mathbb{C}$ is the unique finite-dimensional irreducible $A\left(\widetilde{V}_{k}(\mathfrak{g})\right)$-module. Then $\widetilde{V}_{k}(\mathfrak{g})=V_{k}(\mathfrak{g})$.

Proof. Assume that $\widetilde{V}_{k}(\mathfrak{g})$ is not simple. Then it contains a non-zero graded ideal $I \neq \widetilde{V}_{k}(\mathfrak{g})$ with respect to $L_{\mathfrak{g}}(0)$ :

$$
I=\bigoplus_{n \in \mathbb{Z}_{\geq 0}} I\left(n+n_{0}\right), \quad L_{\mathfrak{g}}(0) \mid I(r)=r \mathrm{Id}, \quad I\left(n_{0}\right) \neq 0 .
$$

Since $I \neq \widetilde{V}_{k}(\mathfrak{g})$, we have that $n_{0}>0$, otherwise $\mathbf{1} \in I$.

We can consider $I\left(n_{0}\right)$ as a finite-dimensional module for $\mathfrak{g}$ and for the Zhu algebra $A\left(\widetilde{V}_{k}(\mathfrak{g})\right)$.

Since the Casimir element $C_{\mathfrak{g}}$ of $\mathfrak{g}$ acts on $I\left(n_{0}\right)$ as the non-zero constant $2\left(k+h^{\vee}\right) n_{0}$, we conclude that $C_{\mathfrak{g}}$ acts by the same constant on any irreducible $\mathfrak{g}$-subquotient of $I\left(n_{0}\right)$. But any irreducible subquotient of $I\left(n_{0}\right)$ is an irreducible finite-dimensional $A\left(\widetilde{V}_{k}(\mathfrak{g})\right)$-module, and therefore it is trivial. This implies that $C_{\mathfrak{g}}$ acts non-trivially on a trivial $\mathfrak{g}$-module, a contradiction.

Take the Chevalley generators $e_{i}, f_{i}, h_{i}, i=0, \ldots, \ell$, of the Kac-Moody Lie algebra $\widehat{\mathfrak{g}}$ such that $e_{i}, f_{i}, h_{i}, i=1, \ldots, \ell$, are the Chevalley generators of $\mathfrak{g}$. Let $\sigma$ be the Chevalley antiautomorphism of $\widehat{\mathfrak{g}}$ defined by

$$
e_{i} \mapsto f_{i}, \quad f_{i} \mapsto e_{i}, h_{i} \mapsto h_{i}, \quad d \mapsto d \quad(i=0, \ldots, \ell)
$$

Assume that $M$ is from the category $\mathcal{O}$ of non-critical level $k$. Then $M$ admits the decomposition into weight spaces $M=\bigoplus_{\mu \in \Omega(M)} M_{\mu}$, where $\Omega(M)$ is the set of weights of $M$ and $\operatorname{dim} M_{\mu}<\infty$ for every $\mu \in \Omega(M)$. For a finite-dimensional vector spaces $U$, let $U^{*}$ denote its dual space. Then we have the contravariant functor $M \mapsto M^{\sigma}$ [20] acting on modules from the category $\mathcal{O}$. Here $M^{\sigma}=\bigoplus_{\mu \in \Omega(M)} M_{\mu}^{*}$ is the $\widehat{\mathfrak{g}}-$ module uniquely determined by

$$
\left\langle y w^{\prime}, w\right\rangle=\left\langle w^{\prime}, \sigma(y) w\right\rangle, \quad y \in \widehat{\mathfrak{g}}, w^{\prime} \in M^{\sigma}, w \in M .
$$

It is easy to see that $M$ admits the decomposition

$$
M=\bigoplus_{\alpha \in \mathbb{C}} M(\alpha), \quad L_{\mathfrak{g}}(0) \mid M(\alpha) \equiv \alpha \operatorname{Id}
$$

such that :

- for any $\alpha \in \mathbb{C}$ we have $M(\alpha-n)=0$ for $n \in \mathbb{Z}$ sufficiently large;

- for any $\mu \in \Omega(M)$ there exist $\alpha \in \mathbb{C}$ such that $M_{\mu} \subset M(\alpha)$. 
Proposition 3.4. Assume that a module $M$ is in the category $\mathcal{O}^{k}$. Then $M$ is in the category $K L^{k}$ if and only if $M$ is $\mathfrak{g}$-locally finite.

Proof. If $M$ is in $K L^{k}$ then it admits a decomposition as in (3.1). Since the spaces $M(\alpha)$ are $\mathfrak{g}$-stable and finite-dimensional, $M$ is $\mathfrak{g}$-locally finite.

Let us prove the converse. If $M$ is a highest weight module which is $\mathfrak{g}$-locally finite, then clearly all eigenspaces for $L_{\mathfrak{g}}(0)$ are finite-dimensional. Assume now that $M$ is an arbitrary $\mathfrak{g}$-locally finite module in the category $\mathcal{O}^{k}$. Take $\alpha \in \mathbb{C}$ such that $M(\alpha) \neq\{0\}$. Then from [20, Proposition 3.1] we see that $M$ has an increasing filtration (possibly infinite)

$$
\{0\}=M_{0} \subset M_{1} \subset \cdots \subset M
$$

such that for every $j \in \mathbb{Z}_{>0}, M_{j} / M_{j-1} \cong \widetilde{L}\left(\lambda_{j}\right)$ is a highest weight $V^{k}(\mathfrak{g})$-module with highest weight $\lambda_{j}$, which is $\mathfrak{g}$-locally finite. Let $h_{\lambda_{j}}$ denotes the lowest conformal weight of $\widetilde{L}\left(\lambda_{j}\right)$. Since the factors $M_{i} / M_{i-1}(i \leq j)$ of $M_{j}$ are highest weight modules, their $L_{\mathfrak{g}}(0)$-eigenspaces are finite-dimensional. This implies that the $L_{\mathfrak{g}}(0)$-eigenspaces of $M_{j}$ is finite-dimensional. By using the properties of the category $\mathcal{O}$ one sees the following:

- There exists a finite subset $\left\{d_{1}, \cdots, d_{s}\right\} \subset \mathbb{C}$ such that $\alpha \in \bigcup_{i=1}^{s}\left(d_{i}+\mathbb{Z}_{\geq 0}\right)$.

- For $d \in \mathbb{C}$ there exist only finitely many subquotients $\widetilde{L}\left(\lambda_{j}\right)$ in (3.2) such that $h_{\lambda_{j}}=d$.

This implies that there is $j_{0} \in \mathbb{Z}_{>0}$ such that $\alpha<h_{\lambda_{j}}$ for $j \geq j_{0}$. Therefore $M(\alpha) \subset M_{j_{0}}$. This proves that $M(\alpha)$ is finite-dimensional.

Remark 3.5. We will use several times the following fact, which is a consequence of the previous proposition: for any $k \notin \mathbb{Z}_{\geq 0}$ and any irreducible highest weight module $L(\lambda)$ in the category $K L^{k}$, one has $\lambda\left(\alpha_{0}^{\vee}\right) \notin \mathbb{Z}_{\geq 0}$.

Since $\sigma\left(L_{\mathfrak{g}}(0)\right)=L_{\mathfrak{g}}(0)$, if $M$ is in the category $K L^{k}$, then $M^{\sigma}$ is also in the category $K L^{k}$. The next result shows that this functor acts on the category $K L_{k}$. In the proof we find an explicit relation of $M^{\sigma}$ with the contragradient modules, defined for ordinary modules for vertex operator algebras 24.

\section{Lemma 3.6.}

(1) Assume that $M$ is a $V_{k}(\mathfrak{g})$-module in the category $\mathcal{O}$. Then $M^{\sigma}$ is also a $V_{k}(\mathfrak{g})$-module in the category $\mathcal{O}$.

(2) Assume that $M$ is a $V_{k}(\mathfrak{g})$-module in the category $K L_{k}$. Then $M^{\sigma}$ is also in $K L_{k}$.

Proof. Assume that $M$ is a $V_{k}(\mathfrak{g})$-module in the category $\mathcal{O}$. Take the weight decomposition $M=$ $\bigoplus_{\mu \in \Omega(M)} M_{\mu}$, and set $M^{c}=\bigoplus_{\mu \in \Omega(M)} M_{\mu}^{*}$. By applying the same approach as in the construction of the contragredient module from [24, Section 5], we get a $V_{k}(\mathfrak{g})$-module $\left(M^{c}, Y_{M^{c}}(\cdot, z)\right)$, with vertex operator map

$$
\left\langle Y_{M^{c}}(v, z) w^{\prime}, w\right\rangle=\left\langle w^{\prime}, Y_{M}\left(e^{z L_{\mathfrak{g}}(1)}\left(-z^{-2}\right)^{L_{\mathfrak{g}}(0)} v, z\right) w\right\rangle,
$$

where $w^{\prime} \in M^{c}, w \in M$. The $\widehat{\mathfrak{g}}_{-}$-action on $M^{c}$ is uniquely determined by

$$
\left\langle x(n) w^{\prime}, w\right\rangle=-\left\langle w^{\prime}, x(-n) w\right\rangle \quad(x \in \mathfrak{g}) .
$$

As a vector space $M^{c}=M^{\sigma}$, but we have different actions of $\widehat{\mathfrak{g}}$. (Note that, in general, $M^{c}$ can be outside of the category $\mathcal{O}$.)

Take the Lie algebra automorphism $h \in A u t(\mathfrak{g})$ such that

$$
e_{i} \mapsto-f_{i}, \quad f_{i} \mapsto-e_{i}, h_{i} \mapsto-h_{i} \quad(i=1, \ldots, \ell) .
$$

Then $h$ can be lifted to an automorphism of $V^{k}(\mathfrak{g})$. Since the maximal ideal of $V^{k}(\mathfrak{g})$ is unique, then it is $h$-invariant, thus $h$ is also an automorphism of $V_{k}(\mathfrak{g})$. Then we can define a $V_{k}(\mathfrak{g})$-module $\left(M_{h}^{c}, Y_{M_{h}^{c}}(\cdot, z)\right)$ where

$$
M_{h}^{c}:=M^{c}, \quad Y_{M_{h}^{c}}(v, z)=Y_{M^{c}}(h v, z) .
$$


On $M_{h}^{c}$ we have

$$
\begin{aligned}
\left\langle e_{i}(n) w^{\prime}, w\right\rangle & =\left\langle w^{\prime}, f_{i}(-n) w\right\rangle \\
\left\langle f_{i}(n) w^{\prime}, w\right\rangle & =\left\langle w^{\prime}, e_{i}(-n) w\right\rangle \\
\left\langle h_{i}(n) w^{\prime}, w\right\rangle & =\left\langle w^{\prime}, h_{i}(-n) w\right\rangle
\end{aligned}
$$

where $i=1, \ldots, \ell$. This implies that $M_{h}^{c}=M^{\sigma}$. This proves the assertion (1).

Assume now that $M$ is in the category $K L_{k}$. Then all $L_{\mathfrak{g}}(0)$-eigenspaces are finite-dimensional, thus

$$
M^{c}=\bigoplus_{\mu \in \Omega(M)} M_{\mu}^{*}=\bigoplus_{\alpha \in \mathbb{C}} M(\alpha)^{*} .
$$

This implies the $V_{k}(\mathfrak{g})$-module $\left(M^{c}, Y_{M^{c}}(\cdot, z)\right)$ coincides with the contragredient module [24, realized on the restricted dual space $\bigoplus_{\alpha \in \mathbb{C}} M(\alpha)^{*}$, with the vertex operator map (3.3). Since the $L_{\mathfrak{g}}(0)$ eigenspaces of $M^{c}$ are finite-dimensional, we conclude that $M^{c}$ and $M^{\sigma}=M_{h}^{c}$ are $V_{k}(\mathfrak{g})$-modules in $K L_{k}$. Claim (2) follows.

\section{Constructions of vertex algebras With one irreducible module in $K L_{k}$ Via COLLAPSING LEVELS}

By 4, if $k$ is a collapsing level, then either $W_{k}(\mathfrak{g}, \theta)=\mathbb{C}, W_{k}(\mathfrak{g}, \theta)=M(1)$, or $W_{k}(\mathfrak{g}, \theta)=V_{k^{\prime}}(\mathfrak{a})$ for a unique simple component $\mathfrak{a}$ of $\mathfrak{g}^{\natural}$. Here the level $k^{\prime}$ is computed with respect to the invariant bilinear form of $\mathfrak{a}$ normalized so that the minimal root has squared length 2 . For $\mathfrak{a}=s l(m \mid n), m \geq 2$, the minimal root is always chosen to be the lowest root of $\operatorname{sl}(m)$. For $\mathfrak{a}=\operatorname{osp}(m \mid n)$ we write $\operatorname{spo}(n \mid m)$ vs. $\operatorname{osp}(m \mid n)$ to specify the choice of the minimal root. In all other cases the minimal root of $\mathfrak{a}$ is unique.

To simplify notation define $V_{k^{\prime}}\left(\mathfrak{g}^{\natural}\right)$ to be as follows:

$$
V_{k^{\prime}}\left(\mathfrak{g}^{\natural}\right)= \begin{cases}\mathbb{C} & \text { if } W_{k}(\mathfrak{g}, \theta)=\mathbb{C} ; \text { in this case we set } k^{\prime}=0 ; \\ M(1) & \text { if } W_{k}(\mathfrak{g}, \theta)=M(1) ; \text { in this case we set } k^{\prime}=1 ; \\ V_{k^{\prime}}(\mathfrak{a}) & \text { otherwise. }\end{cases}
$$

In Table 5 we summarize all the relevant data.

Assume that $k \notin \mathbb{Z}_{\geq 0}$ and that:

(1) $k$ is a collapsing level for $\mathfrak{g}$;

(2) $V_{k^{\prime}}\left(\mathfrak{g}^{\natural}\right)$ is the unique irreducible $V_{k^{\prime}}\left(\mathfrak{g}^{\natural}\right)$-module in the category $K L_{k^{\prime}}$.

Assume that $L(\widehat{\Lambda})$ is an irreducible $V_{k}(\mathfrak{g})$-module in the category $K L_{k}$. Set $\mu=\widehat{\Lambda}_{\mid \mathfrak{h}}$. By Proposition 3.4 we have $\mu \in P_{+}$, hence, by (2.5), the weight $\mu$ has the form $\mu=\mu^{\natural}+\ell \theta$ with $\ell \in \frac{1}{2} \mathbb{Z}_{\geq 0}$, where $\mu^{\natural}=\mu_{\mid \mathfrak{h}}$.

Since $k \notin \mathbb{Z}_{>0}$, by Theorem 2.1 $H_{\theta}(L(\widehat{\Lambda}))$ is a non-trivial irreducible module for $W_{k}(\mathfrak{g}, \theta)$. Since $L(\widehat{\Lambda})$ is a quotient of the Verma module $M(\widehat{\Lambda})$, then, by exactness of $H_{\theta}, H_{\theta}(L(\widehat{\Lambda}))$ is the quotient of a Verma module for $W_{k}(\mathfrak{g}, \theta)=V_{k^{\prime}}\left(\mathfrak{g}^{\natural}\right)$ hence it is an irreducible highest weight module. By [35. (6.14)] its highest weight as $\mathcal{V}_{k}\left(\mathfrak{g}^{\natural}\right)$-module is $\widehat{\Lambda}^{\natural}$ with $\widehat{\Lambda}^{\natural}(K)=k^{\prime}$ and $\widehat{\Lambda}_{\mid \mathfrak{h}^{\natural}}^{\natural}=\mu^{\natural}$. Therefore

$$
H_{\theta}(L(\widehat{\Lambda}))=L_{\mathfrak{g}^{\natural}}\left(\widehat{\Lambda}^{\natural}\right) .
$$

In particular $H_{\theta}(L(\widehat{\Lambda}))$ is in the category $K L_{k^{\prime}}$.

Moreover, under the identification of the centralizer $\mathfrak{g}^{f}$ of $f$ in $\mathfrak{g}$ with $\mathfrak{g}_{0} \oplus \mathfrak{g}_{1 / 2}$ via $\operatorname{ad}(f)$ (see Example 6.2 of [35]), we get that $x$ acts on $H_{\theta}(L(\widehat{\Lambda}))$ via $J_{0}^{\{f\}}$, and $J^{\{f\}}$ is the conformal vector of $W(k, \theta)$ (see the proof of Theorem 5.1 of [35]). Since the level is collapsing we know, by Proposition 4.1 of [4], that the conformal vector of $W_{k}(\mathfrak{g}, \theta)$ coincides with the Segal-Sugawara vector conformal 
Table 5

Values of $k$ and $k^{\prime}$.

\begin{tabular}{|c|c|c|c|}
\hline $\mathfrak{g}$ & $V_{k^{\prime}}\left(\mathfrak{g}^{\mathfrak{h}}\right)$ & $k$ & $k^{\prime}$ \\
\hline$s l(m \mid n), m \neq n, m>3, m-2 \neq n$ & $\left.V_{k^{\prime}}(\operatorname{sl}(m-2 \mid n))\right)$ & $\frac{n-m}{2}$ & $\frac{n-m+2}{2}$ \\
\hline $\operatorname{sl}(3 \mid n), n \neq 3, n \neq 1, n \neq 0$ & $\left.V_{k^{\prime}}(\operatorname{sl}(1 \mid n))\right)$ & $\frac{n-3}{2}$ & $\frac{1-n}{2}$ \\
\hline $\operatorname{sl}(3)$ & $\mathbb{C}$ & $-\frac{3}{2}$ & 0 \\
\hline$s l(2 \mid n), n \neq 2, n \neq 1, n \neq 0$ & $\left.V_{k^{\prime}}(\operatorname{sl}(n))\right)$ & $\frac{n-2}{2}$ & $-\frac{n}{2}$ \\
\hline$s l(2 \mid 1)=\operatorname{spo}(2 \mid 2)$ & $\mathbb{C}$ & $-\frac{1}{2}$ & 0 \\
\hline$s l(m \mid n), m \neq n, n+1, n+2, m \geq 2$ & $M(1)$ & -1 & 1 \\
\hline $\operatorname{psl}(m \mid m), m \geq 2$ & $\mathbb{C}$ & -1 & 0 \\
\hline $\operatorname{spo}(n \mid m), m \neq n, n+2, n \geq 4$ & $V_{k^{\prime}}(\operatorname{spo}(n-2 \mid m))$ & $\frac{m-n-4}{4}$ & $\frac{m-n-2}{4}$ \\
\hline $\operatorname{spo}(2 \mid m), m \geq 5$ & $V_{k^{\prime}}(s o(m))$ & $\frac{m-6}{4}$ & $\frac{4-m}{2}$ \\
\hline $\operatorname{spo}(2 \mid 3)$ & $V_{k^{\prime}}(\operatorname{sl}(2))$ & $-\frac{3}{4}$ & 1 \\
\hline $\operatorname{spo}(2 \mid 1)$ & $\mathbb{C}$ & $-\frac{5}{4}$ & 0 \\
\hline $\operatorname{spo}(n \mid m), m \neq n+1, n \geq 2$ & $\mathbb{C}$ & $-1 / 2$ & 0 \\
\hline$o s p(m \mid n), m \neq n, m \neq n+8, m \geq 7$ & $V_{k^{\prime}}(\operatorname{osp}(m-4 \mid n))$ & $\frac{n-m+4}{2}$ & $\frac{8-m+n}{2}$ \\
\hline $\operatorname{osp}(m \mid n), n \neq m, 0 ; 4 \leq m \leq 6$ & $V_{k^{\prime}}(\operatorname{osp}(m-4 \mid n))$ & $\frac{n-m+4}{2}$ & $\frac{m-n-8}{4}$ \\
\hline$o s p(m \mid n), m \neq n+4, n+8 ; m \geq 4$ & $V_{k^{\prime}}(\operatorname{sl}(2))$ & -2 & $\frac{m-n-8}{2}$ \\
\hline$o s p(n+8 \mid n), n \geq 0$ & $\mathbb{C}$ & -2 & 0 \\
\hline$D(2,1 ; a)$ & $V_{k^{\prime}}(\operatorname{sl}(2))$ & $a$ & $-\frac{1+2 a}{1+a}$ \\
\hline$D(2,1 ; a)$ & $V_{k^{\prime}}(\operatorname{sl}(2))$ & $-a-1$ & $-\frac{1+2 a}{a}$ \\
\hline$F(4)$ & $V_{k^{\prime}}(D(2,1 ; 2))$ & -1 & $\frac{1}{2}$ \\
\hline$F(4)$ & $\mathbb{C}$ & $-3 / 2$ & 0 \\
\hline$F(4)$ & $V_{k^{\prime}}(s o(7))$ & $\frac{2}{3}$ & -2 \\
\hline$F(4)$ & $\mathbb{C}$ & $-\frac{2}{3}$ & 0 \\
\hline$E_{6}$ & $V_{k^{\prime}}(\operatorname{sl}(6))$ & -4 & -1 \\
\hline$E_{6}$ & $\mathbb{C}$ & -3 & 0 \\
\hline$E_{7}$ & $V_{k^{\prime}}(s o(12))$ & -6 & -2 \\
\hline$E_{7}$ & $\mathbb{C}$ & -4 & 0 \\
\hline$E_{8}$ & $V_{k^{\prime}}\left(E_{7}\right)$ & -10 & -4 \\
\hline$E_{8}$ & $\mathbb{C}$ & -6 & 0 \\
\hline$F_{4}$ & $V_{k^{\prime}}(\operatorname{sp}(6))$ & -3 & $-\frac{1}{2}$ \\
\hline$F_{4}$ & $\mathbb{C}$ & $-5 / 2$ & 0 \\
\hline$G_{2}$ & $V_{k^{\prime}}(\operatorname{sl}(2))$ & $-\frac{4}{3}$ & 1 \\
\hline$G_{2}$ & $\mathbb{C}$ & $-\frac{5}{3}$ & 0 \\
\hline$G(3)$ & $V_{k^{\prime}}\left(G_{2}\right)$ & $\frac{1}{2}$ & $-\frac{5}{3}$ \\
\hline$G(3)$ & $\mathbb{C}$ & $-\frac{3}{4}$ & 0 \\
\hline$G(3)$ & $V_{k^{\prime}}(\operatorname{osp}(3 \mid 2))$ & $-\frac{2}{3}$ & 1 \\
\hline$G(3)$ & $\mathbb{C}$ & $-\frac{4}{3}$ & 0 \\
\hline
\end{tabular}


$\omega_{\mathfrak{g}^{\natural}}$ of $V_{k^{\prime}}\left(\mathfrak{g}^{\natural}\right)$ hence, by (6.14) of [35] again, we obtain that the $\left(\omega_{\mathfrak{g}^{\natural}}\right)_{0}$ acts on the lowest component of $H_{\theta}(L(\widehat{\Lambda}))$ by $c I$ with

$$
c=\frac{(\mu+2 \rho, \mu)}{2\left(k+h^{\vee}\right)}-\mu(x) .
$$

Now condition (2) implies that $\mu^{\natural}=0$, so $\mu=\ell \theta$ and

$$
\frac{(\mu+2 \rho, \mu)}{2\left(k+h^{\vee}\right)}-\mu(x)=\frac{(\ell \theta+2 \rho, \ell \theta)}{2\left(k+h^{\vee}\right)}-\ell=0 .
$$

By using formula (2.4), we get

$$
\frac{2 \ell^{2}+\left(2 h^{\vee}-2\right) \ell}{2\left(k+h^{\vee}\right)}-\ell=\frac{\ell^{2}-(k+1) \ell}{k+h^{\vee}}=0 .
$$

- Consider first the case $k=-h^{\vee} / 2+1$ (this holds for $\mathfrak{g}=D_{2 n}, n \geq 2$ and $\mathfrak{g}=\operatorname{osp}(n+4 m+8 \mid n)$, $n \geq 0$ ). Then (4.2) gives that

$$
\frac{2 \ell^{2}+\left(h^{\vee}-4\right) \ell}{h^{\vee}+2}=0 .
$$

We get $\ell=0$ or $2 \ell+h^{\vee}-4=0$.

- Next we consider the case $k=-h^{\vee} / 6-1$. We get

$$
\frac{6 \ell^{2}+h^{\vee} \ell}{5 h^{\vee}-6}=0
$$

We conclude that $\ell=0$ or $\ell=-\frac{h^{\vee}}{6}$.

By using the above analysis and properties of Hamiltonian reduction, we get the following lemma, which extends a result of [15] for Lie algebras to the super case.

Lemma 4.1. Assume that $k=-\frac{h^{\vee}}{6}-1$ and $\mathfrak{g}$ is one of the Lie algebras of exceptional Deligne's series $A_{2}, G_{2}, D_{4}, F_{4}, E_{6}, E_{7}, E_{8}$, or $\mathfrak{g}=\operatorname{psl}(m \mid m)(m \geq 2)$, osp $(n+8 \mid n)(n \geq 2), \operatorname{spo}(2 \mid 1), F(4)$, $G(3)$ (for both choices of $\theta$ ).

Assume that $L(\lambda)$ is a $V_{k}(\mathfrak{g})$-module in the category $\mathcal{O}$. Then one of the following condition holds:

(1) $\lambda\left(\alpha_{0}^{\vee}\right) \in \mathbb{Z}_{\geq 0}$;

(2) $\bar{\lambda}$ is either 0 or $\frac{-h^{\vee}}{6} \theta$, where $\bar{\lambda}$ is the restriction of $\lambda$ to $\mathfrak{h}$.

Proof. By Theorem 2.1 if $L(\lambda)$ is a $V_{k}(\mathfrak{g})$-module for which $\lambda\left(\alpha_{0}^{\vee}\right) \notin \mathbb{Z}_{\geq 0}$, then $H_{\theta}(L(\lambda))$ is an irreducible $W_{k}(\mathfrak{g}, \theta)=H_{\theta}\left(V_{k}(\mathfrak{g})\right)$-module. The conditions on $\mathfrak{g}$ exactly correspond to the cases when $W_{k}(\mathfrak{g}, \theta)$ is one-dimensional (cf. 4], 15]), so the discussion that precedes the Lemma and relation (4.4) imply that $\bar{\lambda}$ is as in (2).

Lemma 4.1 implies:

Theorem 4.2. Assume that the level $k$ and the Lie superalgebra $\mathfrak{g}$ satisfy one of the following conditions:

(1) $k=-\frac{h^{\vee}}{6}-1$ and $\mathfrak{g}$ is one of the Lie algebras of exceptional Deligne's series $A_{2}, G_{2}, D_{4}, F_{4}$, $E_{6}, E_{7}, E_{8}$, or $\mathfrak{g}=\operatorname{psl}(m \mid m)(m \geq 2), \operatorname{osp}(n+8 \mid n)(n \geq 2), \operatorname{spo}(2 \mid 1), F(4), G(3)$ (for both choices of $\theta)$;

(2) $k=-h^{\vee} / 2+1$ and $\mathfrak{g}=\operatorname{osp}(n+4 m+8 \mid n), n \geq 2, m \geq 0$.

(3) $k=-h^{\vee} / 2+1$ and $\mathfrak{g}=D_{2 m}, m \geq 2$.

(4) $k=-10$ and $\mathfrak{g}=E_{8}$.

Then $V_{k}(\mathfrak{g})$ is the unique irreducible $V_{k}(\mathfrak{g})$-module in the category $K L_{k}$.

Proof. If the Lie superalgebra $\mathfrak{g}$ is as in (1), then Lemma4.1 and Remark 3.5 imply that $\bar{\lambda}$ is either 0 or $\frac{-h^{\vee}}{6} \theta$. Since in all cases in (1) we have that $h^{\vee} \in \mathbb{Z}_{\geq 0}$, one obtains that the irreducible highest weight $\mathfrak{g}$-module with highest weight $\bar{\lambda}=\frac{-h^{\vee}}{6} \theta$ cannot be finite-dimensional. Therefore $L(\lambda)$ can not be a 
module in $K L_{k}$. This proves that $\bar{\lambda}=0$ and therefore $V_{k}(\mathfrak{g})$ is the unique irreducible $V_{k}(\mathfrak{g})$-module in the category $K L_{k}$.

Let us consider the case $\mathfrak{g}=\operatorname{osp}(n+4 m+8 \mid n)$. Then for every $m \in \mathbb{Z}_{\geq 0}$ we have:

$$
\begin{aligned}
& h^{\vee}=4 m+6, \\
& k=-h^{\vee} / 2+1=-2(m+1), \\
& 2 \ell+h^{\vee}-4 \neq 0 \quad \forall \ell \in \frac{1}{2} \mathbb{Z}_{\geq 0} .
\end{aligned}
$$

We prove the claim by induction. In the case $m=0$, the claim was proved in (1). Assume now that the claim holds for $\mathfrak{g}^{\prime}=\operatorname{osp}(n+4(m-1)+8, n)$, and $k^{\prime}=-2 m$.

By Theorem 2.3, $k=-2(m+1)$ is a collapsing level and $W_{k}(\mathfrak{g}, \theta)=V_{k^{\prime}}\left(\mathfrak{g}^{\prime}\right)$.

By inductive assumption $V_{k^{\prime}}\left(\mathfrak{g}^{\prime}\right)$ is the unique irreducible $V_{k^{\prime}}\left(\mathfrak{g}^{\prime}\right)$ in the category $K L_{k^{\prime}}$. By applying (4.3) and (4.7) we get that $\ell=0$ and therefore $V_{k}(\mathfrak{g})$ is the unique irreducible $V_{k}(\mathfrak{g})$-module in the category $K L_{k}$. The assertion now follows by induction on $m$.

(3) is a special case of (2), by taking $n=0$.

(4) follows from the fact that $H_{\theta}\left(V_{-10}\left(E_{8}\right)\right)=V_{-4}\left(E_{7}\right)$ and case (1) by applying formula (4.2).

Remark 4.3. Theorem 4.2 can be also proved by non-cohomological methods, using explicit formulas for singular vectors and Zhu algebra theory. As an illustration, we shall present in Theorem 8.6 a direct proof in the case of $D_{2 n}$ at level $k=-h^{\vee} / 2+1$.

In the following sections we shall study some other applications of collapsing levels. We shall restrict our analysis to the case of Lie algebras. In what follows we let $\omega_{1}, \ldots, \omega_{n}$ be the fundamental weights for $\mathfrak{g}$ and $\Lambda_{0}, \ldots, \Lambda_{n}$ the fundamental weights for $\widehat{\mathfrak{g}}$.

\section{On COMPlete REDUCiBility in the CATEgory $K L_{k}$}

In this Section we prove complete reducibility results in the category $K L_{k}$ when $\mathfrak{g}$ is a Lie algebra. We start with a preliminary result, which also holds in the super setting.

Lemma 5.1. Assume that the Lie superalgebra $\mathfrak{g}$ and level $k$ satisfy the conditions of Theorem 4.2. Assume that $M$ is a highest weight $V_{k}(\mathfrak{g})$-module from the category $K L_{k}$. Then $M$ is irreducible.

Proof. By using the classification of irreducible modules from Theorem 4.2 we know that the highest weight of $M$ is necessary $k \Lambda_{0}$, and therefore $M$ is a $\mathbb{Z}_{\geq 0}$-graded with respect to $L_{\mathfrak{g}}(0)$. Denote a highest weight vector by $w_{k \Lambda_{0}}$. We have that

$$
L_{\mathfrak{g}}(0) v=0 \quad \Longleftrightarrow \quad v=\nu w_{k \Lambda_{0}} \quad(\nu \in \mathbb{C}) .
$$

Assume that $M$ is not irreducible. Then it contains a non-zero graded submodule $N \neq M$ with respect to $L_{\mathfrak{g}}(0)$ :

$$
N=\bigoplus_{n \in \mathbb{Z}_{\geq 0}} N\left(n+n_{0}\right), \quad L_{\mathfrak{g}}(0)_{\mid N(r)}=r \mathrm{Id}, \quad N\left(n_{0}\right) \neq 0 .
$$

Since $N \neq M$, we have that $n_{0}>0$, otherwise $w_{k \Lambda_{0}} \in M$.

We can consider $N\left(n_{0}\right)$ as a finite-dimensional module for $\mathfrak{g}$ and for the Zhu algebra $A\left(V_{k}(\mathfrak{g})\right)$. Note that Theorem 4.2 and Proposition 3.2 imply that any irreducible finite-dimensional $A\left(V_{k}(\mathfrak{g})\right)$-module is trivial. Since the Casimir element $C_{\mathfrak{g}}$ of $\mathfrak{g}$ acts on $N\left(n_{0}\right)$ as the non-zero constant $2\left(k+h^{\vee}\right) n_{0}$, we conclude that $C_{\mathfrak{g}}$ acts by the same constant on any irreducible $\mathfrak{g}$-subquotient of $N\left(n_{0}\right)$. But any irreducible subquotient of $N\left(n_{0}\right)$ is an irreducible finite-dimensional $A\left(V_{k}(\mathfrak{g})\right)$-module, and therefore it is trivial. This implies that $C_{\mathfrak{g}}$ acts non-trivially on a trivial $\mathfrak{g}$-module, a contradiction.

The following Lemma is a consequence of [28, Theorem 0.1].

Lemma 5.2. 28] Assume that $\mathfrak{g}$ is a simple Lie algebra and $k$ is a rational number, $k>-h^{\vee}$. Then, in the category of $V_{k}(\mathfrak{g})$-modules, we have: $\operatorname{Ext}^{1}\left(V_{k}(\mathfrak{g}), V_{k}(\mathfrak{g})\right)=(0)$. 
Theorem 5.3. Assume that $\mathfrak{g}$ is a simple Lie algebra and that the level $k$ satisfies the conditions of Theorem 4.2. Then any $V_{k}(\mathfrak{g})$-module $M$ from the category $K L_{k}$ is completely reducible.

Proof. Since $M$ is in $K L_{k}$ we have that any irreducible subquotient of $M$ is isomorphic to $V_{k}(\mathfrak{g}) . M$ has finite length. This implies that $M$ is $\mathbb{Z}_{\geq 0}$-graded:

$$
M=\bigoplus_{n \in \mathbb{Z} \geq 0} M(n), \quad L_{\mathfrak{g}}(0)_{\mid M(r)}=r \mathrm{Id} .
$$

Assume that $M(0)=\operatorname{span}_{\mathbb{C}}\left\{w_{1}, \ldots, w_{s}\right\}$. Then by Lemma 5.1 we have that $V_{k}(\mathfrak{g}) w_{i} \cong V_{k}(\mathfrak{g})$ for every $i=1, \ldots, s$. Now using Lemma 5.2 we get $M \cong \oplus V_{k}(\mathfrak{g}) w_{i}$ and therefore $M$ is completely reducible.

Remark 5.4. We expect that the previous theorem holds in the case when $\mathfrak{g}$ is the Lie superalgebra from Theorem 4.2. We shall study this case in 7 .

We shall now prove much more general result on complete reducibility in $K L_{k}$.

Theorem 5.5. Assume that level $k \in \mathbb{Q}, k>-h^{\vee}$, and the simple Lie algebra $\mathfrak{g}$ satisfy the following property:

$$
\text { Every highest weight } V_{k}(\mathfrak{g}) \text {-module in } K L_{k} \text { is irreducible. }
$$

Then the category $K L_{k}$ is semi-simple.

Proof. We shall present a sketch of the proof and omit some standard representation theoretic arguments which can be found in 20] and 28].

- Since every irreducible $V_{k}(\mathfrak{g})$-module in $K L_{k}$ is isomorphic to $L(\lambda)$ for certain rational, noncritical weight $\lambda$, then [28, Theorem 0.1] implies that $\operatorname{Ext}^{1}(L(\lambda), L(\lambda))=(0)$ in the category $K L_{k}$.

- We prove that in the category $K L_{k}$ we have

$$
\operatorname{Ext}^{1}\left(L_{1}, L_{2}\right)=(0)
$$

for any two irreducible modules $L_{1}$ and $L_{2}$ from $K L_{k}$.

It remains to consider the case $L_{1} \neq L_{2}$. Take an exact sequence in $K L_{k}$ :

$$
0 \rightarrow L\left(\lambda_{1}\right) \rightarrow M \rightarrow L\left(\lambda_{2}\right) \rightarrow 0,
$$

where $\lambda_{1} \neq \lambda_{2}$. Then $M$ contains a singular vector $w_{\lambda_{1}}$ of highest weight $\lambda_{1}$ and a subsingular vector $w_{\lambda_{2}}$ of weight $\lambda_{2}$ and $w_{\lambda_{1}}$ generates a submodule isomorphic to $L\left(\lambda_{1}\right)$. Consider the case $\lambda_{1}-\lambda_{2} \notin Q_{+}$. Then $\lambda_{2}$ is a maximal element of the set $\Omega(M)$ of weights of $M$, and therefore the subsingular vector $w_{\lambda_{2}}$ in $M$ of weight $\lambda_{2}$ is a singular vector. By (5.1), it generates an irreducible module isomorphic to $L\left(\lambda_{2}\right)$ and we conclude that $M \cong L\left(\lambda_{1}\right) \oplus L\left(\lambda_{2}\right)$.

If $\lambda_{1}-\lambda_{2} \in Q_{+}$we can use the contravariant functor $M \mapsto M^{\sigma}$ and get an exact sequence

$$
0 \rightarrow L\left(\lambda_{2}\right) \rightarrow M^{\sigma} \rightarrow L\left(\lambda_{1}\right) \rightarrow 0 .
$$

Since $M^{\sigma}$ is again a $V_{k}(\mathfrak{g})$-module in $K L_{k}$ (cf. Lemma 3.6) by the first case we have that $M^{\sigma}=L\left(\lambda_{1}\right) \oplus L\left(\lambda_{2}\right)$. This implies that

$$
M=L\left(\lambda_{1}\right)^{\sigma} \oplus L\left(\lambda_{2}\right)^{\sigma}=L\left(\lambda_{1}\right) \oplus L\left(\lambda_{2}\right) .
$$

- Assume now that $M$ is a finitely generated module from $K L_{k}$. Then from 20, Proposition 3.1] we see that $M$ has an increasing filtration

$$
(0)=M_{0} \subseteq M_{1} \subseteq \cdots
$$

such that

(1) for every $j \in \mathbb{Z}_{>0}, M_{j} / M_{j-1}$ is an highest weight module in category $\mathcal{O}$;

(2) for any weight $\lambda$ of $M$, there exists $r$ such that $\left(M / M_{r}\right)_{\lambda}=0$. 
Since $M$ is finitely generated as $\widehat{\mathfrak{g}}$-module, we can assume that its generators are weight vectors of weights say $\mu_{1}, \ldots \mu_{p}$. Since they are a finite number there certainly exists $t$ such that $\left(M / M_{t}\right)_{\mu_{i}}=0$ for all $i=1, . ., p$. Hence the filtration (5.3) is finite and stops at $M=M_{t}$. Since $M$ is in category $K L_{k}$, we have that the factors of (5.3) are in category $K L_{k}$. Hence, by our assumption, they are irreducible. Therefore (5.3) is a composition series of finite length. Using assumption (5.1), relation (5.2) and induction on $t$ we get that

$$
M \cong \bigoplus_{j=1}^{t} L\left(\lambda_{j}\right) .
$$

- Finally, we shall consider the case when $M$ is not finitely generated. Since $M$ is in $K L_{k}$, it is countably generated. So $M=\cup_{n=1}^{\infty} M^{(n)}$ such that each $M^{(n)}$ is finitely generated $V_{k}(\mathfrak{g})$-module. By previous case $M^{(n)}$ is completely reducible, so:

$$
M^{(n)}=\bigoplus_{i=1}^{n_{i}} L\left(\lambda_{i, n}\right) .
$$

Therefore $M$ is a sum of irreducible modules from $K L_{k}$ and by using classical algebraic arguments one can see that $M$ is a direct sum of countably many irreducible modules from $K L_{k}$ appearing in decompositions (5.4).

The claim follows.

In order to apply Theorem 5.5, the basic step is to check relation (5.1). We have the following method.

Lemma 5.6. Let $k \in \mathbb{Q} \backslash \mathbb{Z}_{\geq 0}$. Assume that $H_{\theta}(U)$ is an irreducible, non-zero $W_{k}(\mathfrak{g}, \theta)=H_{\theta}\left(V_{k}(\mathfrak{g})\right)$ module for every non-zero highest weight $V_{k}(\mathfrak{g})$-module $U$ from the category $K L_{k}$. Then every highest weight $V_{k}(\mathfrak{g})$-module in $K L_{k}$ is irreducible.

Proof. Assume that $M$ is a highest weight $V_{k}(\mathfrak{g})$-module in $K L_{k}$. Then $H_{\theta}(M)$ is an irreducible $H_{\theta}\left(V_{k}(\mathfrak{g})\right)$-module. If $M$ is not irreducible, then it contains a highest weight submodule $U$ such that $\{0\} \varsubsetneqq U \varsubsetneqq M$. Modules $U$ and $M / U$ are again highest weight modules in $K L_{k}$. By the assumption of the Lemma we have that $H_{\theta}(U)$ is a non-trivial submodule of $H_{\theta}(M)$. Irreducibility of $H_{\theta}(M)$ implies that $H_{\theta}(U)=H_{\theta}(M)$, and therefore $H_{\theta}(M / U)=\{0\}$, a contradiction.

Theorem 5.7. Assume that $\mathfrak{g}$ is a simple Lie algebra and $k \in \mathbb{C} \backslash \mathbb{Z}_{\geq 0}$ such that $W_{k}(\mathfrak{g}, \theta)$ is rational. Then $K L_{k}$ is a semi-simple category.

Proof. Assume that $\widetilde{L}(\lambda)$ is a highest weight $V_{k}(\mathfrak{g})$-module in $K L_{k}$. Clearly $\lambda\left(\alpha_{0}^{\vee}\right) \notin \mathbb{Z}_{\geq 0}$ and by Theorem $2.1 H_{\theta}(\widetilde{L}(\lambda)) \neq(0)$. Since $H_{\theta}(\widetilde{L}(\lambda))$ is non-zero highest weight module for the rational vertex algebra $W_{k}(\mathfrak{g}, \theta)$, we conclude that $H_{\theta}(\widetilde{L}(\lambda))$ is irreducible. Now assertion follows from Theorem 5.5 and Lemma 5.6 .

Remark 5.8. The previous theorem proves that the category $K L_{k}$ is semisimple in the following (non-admissible) cases:

- $\mathfrak{g}=D_{4}, E_{6}, E_{7}, E_{8}$ and $k=-\frac{h^{\vee}}{6}$ using results from [38].

Moreover, using Theorem 5.5 and Lemma 5.6 we can prove the semi-simplicity of $K L_{k}$ for all collapsing levels not accounted by Theorem 1.1. We list here only non-admissible levels, since in admissible case $K L_{k}$ is semi-simple by 12 .

Theorem 5.9. The category $K L_{k}$ is semisimple in the following cases:

(1) $\mathfrak{g}=D_{\ell}, \ell \geq 3$ and $k=-2$;

(2) $\mathfrak{g}=B_{\ell}, \ell \geq 2$ and $k=-2$;

(3) $\mathfrak{g}=A_{\ell}, \ell \geq 2$ and $k=-1$;

(4) $\mathfrak{g}=A_{2 \ell-1}, \ell \geq 2, k=-\ell$; 
(5) $\mathfrak{g}=D_{2 \ell-1}, \ell \geq 3$ and $k=-2 \ell+3$;

(6) $\mathfrak{g}=C_{\ell}, k=-1-\ell / 2$;

(7) $\mathfrak{g}=E_{6}, k=-4$;

(8) $\mathfrak{g}=E_{7}, k=-6$;

(9) $\mathfrak{g}=F_{4}, k=-3$.

Proof. We will give a proof of relations (1) and (2) in Corollaries 6.8 and 7.7 respectively. Case (1) for $\ell \neq 3$ will follow from Theorem 5.7. Note also that case (1) for $\ell=3$ is a special case of case (4), and that case $(2)$ for $\ell=2$ is a special case of (6). The proof in cases (3) - (6) is similar, and it uses the classification of irreducible modules from [10, [11, [16] and the results on collapsing levels [4. Cases $(7)-(9)$ are reduced to cases we have already treated. Here are some details.

Case (3):

- [16], 4] $H_{\theta}\left(V_{-1}\left(A_{\ell}\right)\right)$ is isomorphic to the Heisenberg vertex algebra $M(1)$ of central charge $c=1$

- By using the fact that every highest weight $M(1)$-module is irreducible, we see that if $U$ is a highest weight $V_{-1}\left(A_{\ell}\right)$-module in $K L_{-1}$, then $H_{\theta}(U)$ is a non-trivial irreducible $M(1)-$ module.

Case (4):

- 16], 4] $H_{\theta}\left(V_{-\ell}\left(A_{2 \ell-1}\right)\right)=V_{-\ell+1}\left(A_{2 \ell-3}\right)$.

- For $\ell=2$, we have that every highest weight $V_{-\ell+1}\left(A_{2 \ell-3}\right)=V_{-1}(\operatorname{sl}(2))$-module $\widetilde{L}(\lambda)$ in $K L_{-1}$ with highest weight $\lambda=-(1+j) \Lambda_{0}+j \Lambda_{1}, j \in \mathbb{Z}_{\geq 0}$, is irreducible.

- By induction, we see that for every highest weight $V_{-\ell}\left(\bar{A}_{2 \ell-1}\right)$-module $U$ in $K L_{-\ell}, H_{\theta}(U)$ is a non-trivial irreducible $V_{-\ell+1}\left(A_{2 \ell-3}\right)$-module.

Case (5)

- $H_{\theta}\left(V_{-2 \ell+3}\left(D_{2 \ell-1}\right)\right) \cong V_{-2 \ell+5}\left(D_{2 \ell-3}\right)$.

- By induction we see that for or every highest weight $V_{-2 \ell+3}\left(D_{2 \ell-1}\right)$-module $U$ in $K L_{-2 \ell+3}$, $H_{\theta}(U)$ is a non-trivial irreducible $V_{-2 \ell+5}\left(D_{2 \ell-3}\right)$-module.

Case (6)

- $H_{\theta}\left(V_{-1-\ell / 2}\left(C_{\ell}\right)\right) \cong V_{-1 / 2-\ell / 2}\left(C_{\ell-1}\right)$.

- For $\ell=2$, we have that every highest weight $V_{-1 / 2-\ell / 2}\left(C_{\ell-1}\right)=V_{-3 / 2}(s l(2))$-module in $K L_{-3 / 2}$ is irreducible.

- By induction, we see that for every highest weight $V_{-1-\ell / 2}\left(C_{\ell}\right)$-module $U$ in $K L_{-1-\ell / 2}, H_{\theta}(U)$ is a non-trivial irreducible $V_{-1 / 2-\ell / 2}\left(C_{\ell-1}\right)$-module.

The proof follows by applying Theorem 5.5 and Lemma 5.6.

Cases $(7)-(8)$

We have

$$
H_{\theta}\left(V_{-4}\left(E_{6}\right)\right)=V_{-1}\left(A_{3}\right), \quad H_{\theta}\left(V_{-6}\left(E_{7}\right)\right)=V_{-2}\left(D_{6}\right),
$$

and these cases are settled in (3) and Theorem 1.1 (3) respectively. Case (9) follows from the fact that $H_{\theta}\left(V_{-3}\left(F_{4}\right)\right)$ is isomorphic to the admissible affine vertex algebra $V_{-\frac{1}{2}}\left(C_{3}\right)$ which is semisimple in $K L_{-1 / 2}$ (cf. [1]).

Remark 5.10. The problem of complete-reducibility of modules in $K L_{k}$ when $\mathfrak{g}$ is a Lie superalgebra will be also studied in [7. An important tool in the description of the category $K L_{k}$ will be the conformal embedding of $\widetilde{V}_{k}\left(\mathfrak{g}_{0}\right)$ to $V_{k}(\mathfrak{g})$ where $\mathfrak{g}_{0}$ is the even part of $\mathfrak{g}$.

Note that in the category $\mathcal{O}$ we can have indecomposable $V_{k}(\mathfrak{g})$-modules in some cases listed in Theorem [5.9. See [10, Remark 5.8] for one example. 


\section{The vertex Algebra $V^{-2}\left(D_{\ell}\right)$ And its Quotients}

In this section we exploit Hamiltonian reduction and the results on conformal embeddings from [4] to investigate the quotients of the vertex algebra $V^{-2}\left(D_{\ell}\right)$. In particular we are interested in a non-simple quotient $\mathcal{V}_{-2}\left(D_{\ell}\right)$ which appears in the analysis of certain dual pairs (see [6]) as well as in the simple quotient $V_{-2}\left(D_{\ell}\right)$. We will show that the vertex algebra $\mathcal{V}_{-2}\left(D_{\ell}\right)$ has infinitely many irreducible modules in the category $K L^{-2}$, while by [15], $V_{-2}\left(D_{\ell}\right)$ has finitely many irreducible modules in $K L_{-2}$. Recall that -2 is a collapsing level for $D_{\ell}[4$.

Consider the vector

$$
w_{1}:=\left(e_{\epsilon_{1}+\epsilon_{2}}(-1) e_{\epsilon_{3}+\epsilon_{4}}(-1)-e_{\epsilon_{1}+\epsilon_{3}}(-1) e_{\epsilon_{2}+\epsilon_{4}}(-1)+e_{\epsilon_{1}+\epsilon_{4}}(-1) e_{\epsilon_{2}+\epsilon_{3}}(-1)\right) \mathbf{1} .
$$

It is a singular vector in $V^{-2}\left(D_{\ell}\right)$ (cf. [15]). Note that this vector is contained in the subalgebra $V^{-2}\left(D_{4}\right)$ of $V^{-2}\left(D_{\ell}\right)$.

By using the explicit expression for singular vectors $v_{n}$ in $V^{n-\ell+1}\left(D_{\ell}\right)$ (see (8.1)), we have that

$$
w_{2}:=v_{\ell-3}=\left(\sum_{i=2}^{\ell} e_{\epsilon_{1}-\epsilon_{i}}(-1) e_{\epsilon_{1}+\epsilon_{i}}(-1)\right)^{\ell-3} \mathbf{1}
$$

is a singular vector in $V^{-2}\left(D_{\ell}\right)$.

For $\ell=4$ we also have a third singular vector (cf. [40])

$$
w_{3}:=\left(e_{\epsilon_{1}+\epsilon_{2}}(-1) e_{\epsilon_{3}-\epsilon_{4}}(-1)-e_{\epsilon_{1}+\epsilon_{3}}(-1) e_{\epsilon_{2}-\epsilon_{4}}(-1)+e_{\epsilon_{1}-\epsilon_{4}}(-1) e_{\epsilon_{2}+\epsilon_{3}}(-1)\right) \mathbf{1} .
$$

6.1. The vertex algebra $\mathcal{V}_{-2}\left(D_{\ell}\right)$ for $\ell \geq 4$. Define the vertex algebra

$$
\mathcal{V}_{-2}\left(D_{\ell}\right)=V^{-2}\left(D_{\ell}\right) / J_{\ell}
$$

where

$$
J_{\ell}=\left\langle w_{1}, w_{3}\right\rangle \quad(\ell=4), \quad J_{\ell}=\left\langle w_{1}\right\rangle \quad(\ell \geq 5) .
$$

The following proposition is essentially proven in [6].

\section{Proposition 6.1.}

(1) There is a non-trivial vertex algebra homomorphism $\bar{\Phi}: \mathcal{V}_{-2}\left(D_{\ell}\right) \rightarrow M_{2 \ell}$ where $M_{2 \ell}$ the Weyl vertex algebra of rank $\ell$.

(2) $\mathcal{V}_{-2}\left(D_{\ell}\right)$ is not simple, and $L\left((-2-t) \Lambda_{0}+t \Lambda_{1}\right), t \in \mathbb{Z}_{\geq 0}$ are $\mathcal{V}_{-2}\left(D_{\ell}\right)$-modules.

Proof. The homomorphism $\Phi: V^{-2}\left(D_{\ell}\right) \rightarrow M_{2 \ell}$ was constructed in [6, Section 7]. By direct calculation one proves that $\Phi\left(w_{1}\right)=0$ for $\ell \geq 4$ and $\Phi\left(w_{3}\right)=0$ for $\ell=4$. Finally [6, Lemma 7.1] implies that $L\left((-2-t) \Lambda_{0}+t \Lambda_{1}\right), t \in \mathbb{Z}_{>0}$ are $\mathcal{V}_{-2}\left(D_{\ell}\right)$-modules. Since the simple vertex algebra $V_{-2}\left(D_{\ell}\right)$ has only finitely many irreducible modules in the category $\mathcal{O}\left[15\right.$, we have that $\mathcal{V}_{-2}\left(D_{\ell}\right)$ is not simple.

Next, we exploit the fact that in the case $\mathfrak{g}=D_{\ell}, k=-2$ is a collapsing level, i.e., in the affine $W$-algebra $W^{k}(\mathfrak{g}, \theta)$, all generators $G^{\{u\}}$ at conformal weight $3 / 2, u \in \mathfrak{g}_{-1 / 2}$, belong to the maximal ideal (see 4 for details). This implies that there exists a non-trivial ideal $I$ in $V^{-2}(\mathfrak{g})$ such that $G^{\{u\}} \in H_{\theta}(I)$ for all $u \in \mathfrak{g}_{-1 / 2}$.

Note also that $\mathfrak{g}^{\natural}=A_{1} \oplus D_{\ell-2}$, so we have that $V^{\ell-4}\left(A_{1}\right) \otimes V^{0}\left(D_{\ell-2}\right)$ is a subalgebra of $W^{-2}\left(D_{\ell}, \theta\right)$. In the case $\ell=4$ we identify $D_{2}$ with $A_{1} \oplus A_{1}$.

Lemma 6.2. We have

- $x_{(-1)} \mathbf{1} \in H_{\theta}\left(J_{\ell}\right)$ for all $x \in D_{\ell-2} \subset \mathfrak{g}^{\natural}$,

- $G^{\{u\}} \in H_{\theta}\left(J_{\ell}\right)$ for all $u \in \mathfrak{g}_{-1 / 2}$.

Proof. Assume that $\ell \geq 5$. Since $w_{1}$ is a singular vector in $V^{-2}\left(D_{\ell}\right)$, the ideal $J_{\ell}$ is a highest weight module of highest weight $\lambda=-2 \Lambda_{0}+\epsilon_{1}+\epsilon_{2}+\epsilon_{3}+\epsilon_{4}$. Now, the Main Theorem from 12 implies that $H_{\theta}\left(J_{\ell}\right)$ is a non-trivial highest weight module. By formula [35. (6.14)] the highest weight is $\left(0, \omega_{2}\right)$ and, by (4.1), the conformal weight of its highest weight vector is 1 . Up to a non-zero constant, 
there is only one vector in $W^{-2}\left(D_{\ell}, \theta\right)=V^{\ell-4}\left(A_{1}\right) \otimes V^{0}\left(D_{\ell-2}\right)$ that has these properties, namely $J_{(-1)}^{\left\{e_{\epsilon_{3}+\epsilon_{4}}\right\}} \mathbf{1}$, and therefore $H_{\theta}\left(J_{\ell}\right)$ contains all generators of $V^{0}\left(D_{\ell-2}\right)$.

In the case $\ell=4, w_{1}$ and $w_{3}$ generate submodules $N_{1}$ and $N_{3}$ of highest weights $\lambda_{1}=-2 \Lambda_{0}+\epsilon_{1}+\epsilon_{2}+$ $\epsilon_{3}+\epsilon_{4}, \lambda_{3}=-2 \Lambda_{0}+\epsilon_{1}+\epsilon_{2}+\epsilon_{3}-\epsilon_{4}$, respectively. Applying the same arguments as above we get that $J_{(-1)}^{\left\{e_{\epsilon_{3}} \pm \epsilon_{4}\right\}} \mathbf{1} \in H_{\theta}(I)$, which implies that $H_{\theta}\left(J_{\ell}\right)$ contains all generators of $V^{0}\left(D_{2}\right)=V^{0}\left(A_{1}\right) \otimes V^{0}\left(A_{1}\right)$. Now, claim follows by applying the action of generators of $V^{0}\left(D_{\ell-2}\right)$ to $G^{\{u\}}$ (see [4]).

Proposition 6.3. We have

(1) $H_{\theta}\left(\mathcal{V}_{-2}\left(D_{\ell}\right)\right)=V^{\ell-4}\left(A_{1}\right)$.

(2) $H_{\theta}\left(L\left((-2-t) \Lambda_{0}+t \Lambda_{1}\right)\right) \cong L_{A_{1}}\left((\ell-4-t) \Lambda_{0}+t \Lambda_{1}\right), t \in \mathbb{Z}_{\geq 0}$.

(3) The set $\left\{L\left((-2-t) \Lambda_{0}+t \Lambda_{1}\right) \mid t \in \mathbb{Z}_{\geq 0}\right\}$ provides a complete list of irreducible $\mathcal{V}_{-2}\left(D_{\ell}\right)$-modules from the category $K L^{-2}$.

Proof. By Lemma 6.2 we see that the vertex algebra $H_{\theta}\left(\mathcal{V}_{-2}\left(D_{\ell}\right)\right)$ is generated only by $x_{(-1)} \mathbf{1}, x \in$ $A_{1} \subset D_{\ell}^{\natural}$. So there are only two possibilities: either $H_{\theta}\left(\mathcal{V}_{-2}\left(D_{\ell}\right)\right)=V^{\ell-4}\left(A_{1}\right)$ or $H_{\theta}\left(\mathcal{V}_{-2}\left(D_{\ell}\right)\right)=$ $V_{\ell-4}\left(A_{1}\right)$. Moreover, for every $t \in \mathbb{Z}_{>0}, H_{\theta}\left(L\left((-2-t) \Lambda_{0}+t \Lambda_{1}\right)\right)$ must be the irreducible $H_{\theta}\left(\mathcal{V}_{-2}\left(D_{\ell}\right)\right)-$ module with highest weight $t \omega_{1}$ with respect to $A_{1}$. So $H_{\theta}\left(L\left((-2-t) \Lambda_{0}+t \Lambda_{1}\right)\right) \cong L_{A_{1}}((\ell-4-$ $\left.t) \Lambda_{0}+t \Lambda_{1}\right), t \in \mathbb{Z}_{>0}$. Therefore, $H_{\theta}\left(\mathcal{V}_{-2}\left(D_{\ell}\right)\right)$ contains infinitely many irreducible modules, which gives that $H_{\theta}\left(\mathcal{V}_{-2}\left(D_{\ell}\right)\right)=V^{\ell-4}\left(A_{1}\right)$. In this way we have proved claims (1) and (2).

Let us now prove claim (3).

Assume that $L\left(k \Lambda_{0}+\mu\right)\left(\mu \in P_{+}, k=-2\right)$ is an irreducible $\mathcal{V}_{k}\left(D_{\ell}\right)$-module in the category $K L^{k}$. Then $H_{\theta}\left(L\left(k \Lambda_{0}+\mu\right)\right)$ is a non-trivial irreducible $V^{\ell-4}\left(A_{1}\right)$-module. The representation theory of $V^{\ell-4}\left(A_{1}\right)$ implies that:

$$
H_{\theta}\left(L\left(k \Lambda_{0}+\mu\right)\right)=L_{A_{1}}\left((\ell-4-j) \Lambda_{0}+j \Lambda_{1}\right) \quad \text { for } j \in \mathbb{Z}_{\geq 0} .
$$

Since $D_{\ell}^{\natural}=A_{1} \times D_{\ell-2}$, we conclude that $\mu^{\natural}=j \omega_{1}$ and therefore, by (2.5),

$$
\mu=j \omega_{1}+s \omega_{2}=(s+j) \epsilon_{1}+s \epsilon_{2} \quad\left(s \in \mathbb{Z}_{\geq 0}\right) .
$$

By using the action of $L(0)=\omega_{0}$ on the lowest component of $H_{\theta}\left(L\left(k \Lambda_{0}+\mu\right)\right)$ we get

$$
\frac{(\mu+2 \rho, \mu)}{2\left(k+h^{\vee}\right)}-\mu(x)=\frac{j(j+2)}{4(\ell-2)} \quad\left(x=\theta^{\vee} / 2\right) .
$$

Since $2\left(k+h^{\vee}\right)=2(-2+2 \ell-2)=4(\ell-2)$ and $\mu(x)=(2 s+j) / 2$ we get

$$
(\mu+2 \rho, \mu)-\left(h^{\vee}-2\right)(2 s+j)=j(j+2) .
$$

By direct calculation we get

$$
(\mu+2 \rho, \mu)=(s+j)^{2}+s^{2}+h^{\vee}(s+j)+\left(h^{\vee}-2\right) s,
$$

which gives an equation:

$$
\begin{array}{ll} 
& (s+j)^{2}+s^{2}+h^{\vee}(s+j)+\left(h^{\vee}-2\right) s-\left(h^{\vee}-2\right)(2 s+j)=j(j+2) . \\
\Longleftrightarrow \quad & (s+j)^{2}+s^{2}+h^{\vee}(s+j)-\left(h^{\vee}-2\right)(s+j)=j(j+2) . \\
\Longleftrightarrow \quad & (s+j)(s+j+2)=j(j+2) \\
& \quad s=0 \quad \text { or } \quad s=-2 j-2 .
\end{array}
$$

Since $\mu \in P_{+}$we conclude that $s=0$. Therefore $\mu=j \omega_{1}$ for certain $j \in \mathbb{Z}_{\geq 0}$. The proof of claim (3) is now complete.

6.2. The simple vertex algebra $V_{-2}\left(D_{\ell}\right)$. Next we use the fact that the simple affine $W$-algebra $W_{-2}\left(D_{\ell}, \theta\right)$ is isomorphic to the simple affine vertex algebra $V_{\ell-4}\left(A_{1}\right)$, for $\ell \geq 4$.

Proposition 6.4. The set $\left\{L\left((-2-j) \Lambda_{0}+j \Lambda_{1}\right) \mid j \in \mathbb{Z}_{\geq 0}, j \leq \ell-4\right\}$ provides a complete list of irreducible $V_{-2}\left(D_{\ell}\right)$-modules from the category $K L_{-2}$. 
Proof. Assume that $N$ is an irreducible $V_{-2}\left(D_{\ell}\right)$-module from the category $K L_{-2}$. Then $N$ is also irreducible as $\mathcal{V}_{-2}\left(D_{\ell}\right)$-module, and therefore $N \cong L\left((-2-j) \Lambda_{0}+j \Lambda_{1}\right)$ for certain $j \in \mathbb{Z}_{>0}$. Since $H_{\theta}(N)$ must be an irreducible $H_{\theta}\left(V_{-2}\left(D_{\ell}\right)\right)=W_{-2}\left(D_{\ell}, \theta\right)=V_{\ell-4}\left(A_{1}\right)$-module, we get $j \leq \ell-4$, as desired.

Now we want to describe the maximal ideal in $V^{-2}\left(D_{\ell}\right)$. The next lemma states that any non-trivial ideal in $\mathcal{V}_{-2}\left(D_{\ell}\right)$ is automatically maximal.

Lemma 6.5. Let $\{0\} \neq I \varsubsetneqq \mathcal{V}_{-2}\left(D_{\ell}\right)$ be any non-trivial ideal in $\mathcal{V}_{-2}\left(D_{\ell}\right)$. Then we have

(1) $H_{\theta}(I)$ is the maximal ideal in $V^{\ell-4}\left(A_{1}\right)$.

(2) $I$ is a maximal ideal in $\mathcal{V}_{-2}\left(D_{\ell}\right)$ and $I=L\left(-2(\ell-2) \Lambda_{0}+2(\ell-3) \Lambda_{1}\right)$.

Proof. Assume that $I$ is a non-trivial ideal in $\mathcal{V}_{-2}\left(D_{\ell}\right)$. Then $I$ can be regarded as a $\mathcal{V}_{-2}\left(D_{\ell}\right)$-module in the category $K L^{-2}$ and therefore, by Proposition 6.3 (3), it contains a non-trivial subquotient isomorphic to $L\left((-2-j) \Lambda_{0}+j \Lambda_{1}\right)$ for some $j \in \mathbb{Z}_{\geq 0}$. Since, by part (2) of the aforementioned Proposition, $H_{\theta}\left(L\left((-2-j) \Lambda_{0}+j \Lambda_{1}\right)\right) \neq 0$ for every $j \in \mathbb{Z}_{\geq 0}$, we conclude that $H_{\theta}(I)$ is a non-trivial ideal in $H_{\theta}\left(\mathcal{V}_{-2}\left(D_{\ell}\right)\right)=V^{\ell-4}\left(A_{1}\right)$. But since $V^{\ell-4}\left(A_{1}\right), \ell \geq 4$, contains a unique non-trivial ideal, which is automatically maximal, we have that $H_{\theta}(I)$ is a maximal ideal in $V^{\ell-4}\left(A_{1}\right)$. So

$$
H_{\theta}\left(\mathcal{V}_{-2}\left(D_{\ell}\right) / I\right) \cong V_{\ell-4}\left(A_{1}\right) \text {. }
$$

Assume now that $\mathcal{V}_{-2}\left(D_{\ell}\right) / I$ is not simple. Then it contains a non-trivial singular vector $v^{\prime}$ of weight $-(2+j) \Lambda_{0}+j \Lambda_{1}$ for $j \in \mathbb{Z}_{>0}$. By [12, we have that $H_{\theta}\left(V^{-2}\left(D_{\ell}\right) \cdot v^{\prime}\right)$ is a non-trivial ideal in $V_{\ell-4}\left(A_{1}\right)$ generated by a singular vector of $A_{1}$-weight $j \omega_{1}$. This is a contradiction. So $I$ is the maximal ideal.

Since the maximal ideal in $V^{\ell-4}\left(A_{1}\right)$ is generated by a singular vector of $A_{1}$-weight $2(\ell-3) \omega_{1}$ and since the maximal ideal is simple, we conclude that $I=\mathcal{V}_{-2}\left(D_{\ell}\right) \cdot v_{\text {sing }}$ for a certain singular vector $v_{\text {sing }}$ of weight $\lambda=-2(\ell-2) \Lambda_{0}+2(\ell-3) \Lambda_{1}$. It is also clear that this singular vector is unique, up to scalar factor. Therefore, $I=L\left(-2(\ell-2) \Lambda_{0}+2(\ell-3) \Lambda_{1}\right)$.

Note that in the previous lemma we proved the existence of a singular vector which generates the maximal ideal without presenting a formula for such a singular vector. Since the vector in (6.2) has the correct weight, we also have an explicit expression for this singular vector:

$$
\left(\sum_{i=2}^{\ell} e_{\epsilon_{1}-\epsilon_{i}}(-1) e_{\epsilon_{1}+\epsilon_{i}}(-1)\right)^{\ell-3} \mathbf{1}
$$

\section{Corollary 6.6.}

(1) The maximal ideal in $V^{-2}\left(D_{\ell}\right)$ is generated by the vectors $w_{1}$ and $w_{2}$ for $\ell \geq 5$ and by the vectors $w_{1}, w_{2}, w_{3}$ for $\ell=4$.

(2) The homomorphism $\bar{\Phi}: \mathcal{V}_{-2}\left(D_{\ell}\right) \rightarrow M_{2 \ell}$ is injective. In particular, the vertex algebra $\mathcal{V}_{-2}\left(D_{\ell}\right) \otimes$ $V_{-\ell}\left(A_{1}\right)$ is conformally embedded into $V_{-1 / 2}\left(C_{2 \ell}\right)$.

(3) $\left.\operatorname{ch}\left(\mathcal{V}_{-2}\left(D_{\ell}\right)\right)=\operatorname{ch}\left(V_{-2}\left(D_{\ell}\right)\right)+\operatorname{ch} L(-2(\ell-2)) \Lambda_{0}+2(\ell-3) \Lambda_{1}\right)$.

Remark 6.7. D. Gaiotto in [27] has started a study of the decomposition of $M_{2 \ell}$ as a $V^{-2}\left(D_{\ell}\right) \otimes$ $V_{-\ell}\left(A_{1}\right)$-module in the case $\ell=4$. By combining results from [6. Section 8] and results from this Section we get that

$$
\operatorname{Com}\left(V_{-\ell}\left(A_{1}\right), M_{2 \ell}\right) \cong \mathcal{V}_{-2}\left(D_{\ell}\right) .
$$

So the vertex algebra responsible for the decomposition of $M_{2 \ell}$ is exactly $\mathcal{V}_{-2}\left(D_{\ell}\right)$. Therefore in the decomposition of $M_{2 \ell}$ only modules for $\mathcal{V}_{-2}\left(D_{\ell}\right)$ can appear. In our forthcoming papers we plan to apply the representation theory of $\mathcal{V}_{-2}\left(D_{\ell}\right)$ to the problem of finding branching rules.

Corollary 6.8. For $\ell \geq 3$ the category $K L_{-2}$ is semi-simple.

Proof. The assertion in the case $\ell \geq 4$ follows from Theorem 5.7 since then $W_{-2}\left(D_{\ell}, \theta\right)=V_{\ell-4}(s l(2))$ is a rational vertex algebra.

In the case $\ell=3$, we have that a highest weight $V_{-2}\left(D_{3}\right)$-module $M$ is isomorphic to $\widetilde{L}\left((-2-j) \Lambda_{0}+\right.$ $\left.j \Lambda_{1}\right)$ where $j \in \mathbb{Z}_{\geq 0}$. The irreducibility of $M$ follows easily from the fact that $H_{\theta}(M)$ is isomorphic 
to an irreducible $V_{-1}(s l(2))$-module $\left.L_{A_{1}}(-1-j) \Lambda_{0}+j \Lambda_{1}\right)$. Now claim follows from Theorem 5.5 and Lemma 5.6

\section{The vertex algebra $V^{-2}\left(B_{\ell}\right)$ And its Quotients}

In this section let $\ell \geq 2$. Note that $k=-2$ is a collapsing level for $B_{\ell}$ [4, and that the simple affine $W$-algebra $W_{-2}\left(B_{\ell}, \theta\right)$ is isomorphic to $V_{\ell-\frac{7}{2}}\left(A_{1}\right)$. This implies that $H_{\theta}\left(V_{-2}\left(B_{\ell}\right)\right)=V_{\ell-\frac{7}{2}}\left(A_{1}\right)$. But as in the case of the affine Lie algebra of type $D$, we can construct an intermediate vertex algebra $\mathcal{V}$ so that $H_{\theta}(\mathcal{V})=V^{\ell-7 / 2}\left(A_{1}\right)$.

Remark 7.1. The formula for a singular vector of conformal weight two in $V^{-2}\left(B_{\ell}\right)$ was given in [15. Theorem 4.2] for $\ell \geq 3$, and in [15, Remark 4.3] for $\ell=2$. Note that, for $\ell \geq 4$, the vector $\sigma\left(w_{2}\right)$ from [15] is equal to the vector $w_{1}$ from relation (6.1), i.e. it is contained in the subalgebra $V^{-2}\left(D_{4}\right)$. For $\ell=3$, we have

$$
w_{1}=\left(e_{\epsilon_{1}+\epsilon_{2}}(-1) e_{\epsilon_{3}}(-1)-e_{\epsilon_{1}+\epsilon_{3}}(-1) e_{\epsilon_{2}}(-1)+e_{\epsilon_{1}}(-1) e_{\epsilon_{2}+\epsilon_{3}}(-1)\right) \mathbf{1} .
$$

For $\ell=2$, the singular vector of conformal weight two in $V^{-2}\left(B_{2}\right)$ is equal to

$$
w_{1}=\left(e_{\epsilon_{1}+\epsilon_{2}}(-1) e_{-\epsilon_{2}}(-1)+\frac{1}{2} h_{\epsilon_{2}}(-1) e_{\epsilon_{1}}(-1)-e_{\epsilon_{1}-\epsilon_{2}}(-1) e_{\epsilon_{2}}(-1)\right) \mathbf{1} .
$$

Consider the singular vector in $V^{-2}\left(B_{\ell}\right)$ denoted by $\sigma\left(w_{2}\right)$ in [15, Theorem 4.2] and [17, Section 7]. Let us denote that singular vector by $w_{1}$ in this paper (see Remark 7.1 for explanation).

Then we have the quotient vertex algebra

$$
\mathcal{V}_{-2}\left(B_{\ell}\right)=V^{-2}\left(B_{\ell}\right) /\left\langle w_{1}\right\rangle .
$$

As in the case of the vertex algebra $\mathcal{V}_{-2}\left(D_{\ell}\right)$, we have the non-trivial homomorphism $\mathcal{V}_{-2}\left(B_{\ell}\right) \rightarrow$ $M_{2 \ell+1}$.

The proof of the following result is completely analogous to the proof of Proposition 6.3 and it is therefore omitted.

Proposition 7.2. We have

(1) There is a non-trivial homomorphism $\bar{\Phi}: \mathcal{V}_{-2}\left(B_{\ell}\right) \rightarrow M_{2 \ell+1}$.

(2) $H_{\theta}\left(\mathcal{V}_{-2}\left(B_{\ell}\right)\right)=V^{\ell-7 / 2}\left(A_{1}\right)$.

(3) $H_{\theta}\left(L\left((-2-t) \Lambda_{0}+t \Lambda_{1}\right)\right) \cong L_{A_{1}}\left((\ell-7 / 2-t) \Lambda_{0}+t \Lambda_{1}\right), t \in \mathbb{Z}_{\geq 0}$.

(4) The set

$$
\left\{L\left((-2-t) \Lambda_{0}+t \Lambda_{1}\right) \mid t \in \mathbb{Z}_{\geq 0}\right\}
$$

provides a complete list of irreducible $\mathcal{V}_{-2}\left(B_{\ell}\right)$-modules from the category $K L^{-2}$.

We have the following result on classification of irreducible modules.

Proposition 7.3. Assume that $\ell \geq 3$. Then the set $\left\{L\left((-2-j) \Lambda_{0}+j \Lambda_{1}\right) \mid j \in \mathbb{Z}_{\geq 0}, j \leq 2(\ell-3)+1\right\}$ provides a complete list of irreducible $V_{-2}\left(B_{\ell}\right)$-modules from the category $K L_{-2}$.

Proof. The proof is analogous to the proof of Proposition 6.4 it uses the exactness of the functor $H_{\theta}$ and the representation theory of affine vertex algebras. In particular, we use the result from 8 which gives that the set

$$
\left.\left\{L(-(\ell-7 / 2)-j) \Lambda_{0}+j \Lambda_{1}\right) \mid j \in \mathbb{Z}_{\geq 0}, j \leq 2(\ell-3)+1\right\}
$$

provides a complete list of irreducible $V_{\ell-7 / 2}\left(A_{1}\right)$-modules from the category $K L_{\ell-7 / 2}$.

An important consequence is the simplicity of the vertex algebra $\mathcal{V}_{-2}\left(B_{2}\right)$.

Corollary 7.4. The vertex algebra $\mathcal{V}_{-2}\left(B_{\ell}\right)$ is simple if and only if $\ell=2$. In particular, the set 7.2) provides a complete list of irreducible modules for $V_{-2}\left(B_{2}\right)$ in $K L_{-2}$. 
Proof. Since by Proposition $7.2 \mathcal{V}_{-2}\left(B_{\ell}\right)$ has infinitely many irreducible modules in the category $K L^{-2}$, and, by Proposition $7.3 . V^{-2}\left(B_{\ell}\right)$ has finitely many irreducible modules in the category $K L^{-2}$ (if $\ell \geq 3$ ), we conclude that $\mathcal{V}_{-2}\left(B_{\ell}\right)$ cannot be simple for $\ell \geq 3$.

Let us consider the case $\ell=2$. Assume that $\mathcal{V}_{-2}\left(B_{2}\right)$ is not simple. Then it must contain an ideal $I$ generated by a singular vector of weight $\lambda=(-2-j) \Lambda_{0}+j \Lambda_{1}$ for certain $j>0$. By applying the functor $H_{\theta}$, we get a non-trivial ideal in $V^{-3 / 2}\left(A_{1}\right)$, against the simplicity of $V^{-3 / 2}\left(A_{1}\right)$.

Next we notice that $V^{\ell-7 / 2}\left(A_{1}\right)$ has a unique non-trivial ideal $J$ which is generated by a singular vector of $A_{1}$-weight $2(\ell-2) \omega_{1}$. The ideal $J$ is maximal and simple (cf. [5]). By combining this with properties of the functor $H_{\theta}$ from [12, one proves the existence of a unique maximal ideal $I$ (which is also simple) in $\mathcal{V}_{-2}\left(B_{\ell}\right)$ such that $\left.I \cong L\left(-2(\ell-1) \Lambda_{0}+2(\ell-2) \Lambda_{1}\right)\right)$.

Remark 7.5. The explicit expression for a singular vector which generates $I$ is more complicated that in the case D, and it won't be presented here.

In [6] we constructed a homomorphism $\mathcal{V}_{-2}\left(B_{\ell}\right) \otimes V_{-\ell-1 / 2}\left(A_{1}\right) \rightarrow M_{2 \ell+1}$. The results of this section enable us to find the image of this homomorphism.

Corollary 7.6. We have:

(1) The vertex algebra $\mathcal{V}_{-2}\left(B_{\ell}\right) \otimes V_{-\ell-1 / 2}\left(A_{1}\right)$ is conformally embedded into $V_{-1 / 2}\left(C_{2 \ell+1}\right)$.

(2) The vertex algebra $\mathcal{V}_{-2}\left(B_{\ell}\right)$ for $\ell \geq 3$ contains a unique ideal $I \cong L\left(-2(\ell-1) \Lambda_{0}+2(\ell-2) \Lambda_{1}\right)$ ) and

$$
\operatorname{ch}\left(\mathcal{V}_{-2}\left(B_{\ell}\right)\right)=\operatorname{ch}\left(V_{-2}\left(B_{\ell}\right)\right)+\operatorname{ch}\left(L\left(-2(\ell-1) \Lambda_{0}+2(\ell-2) \Lambda_{1}\right)\right) .
$$

Finally, we apply Theorem 5.5 and prove that $K L_{-2}$ is a semi-simple category.

Corollary 7.7. If $\ell \geq 2$, then every $V_{-2}\left(B_{\ell}\right)$-module in $K L_{-2}$ is completely reducible.

Proof. It suffices to prove that every highest weight $V_{-2}\left(B_{\ell}\right)$-module in $K L_{-2}$ is irreducible. Assume that $\ell \geq 3$. If $M \cong \widetilde{L}(\lambda)$ is a highest weight module in $K L_{-2}$ then the highest weight is $\lambda=-(2+$ $j) \Lambda_{0}+j \Lambda_{1}$ where $0 \leq j \leq 2(\ell-3) j+1$. Since $H_{\theta}(L(\lambda))$ is a non-zero highest weight $V_{-\ell+7 / 2}(s l(2))-$ module, then the complete reducibility result from [8] implies that $H_{\theta}(L(\lambda))$ is irreducible. The assertion now follows from Lemma [5.6. The proof in the case $\ell=2$ is similar, and it uses the classification of irreducible $V_{-2}\left(B_{2}\right)$-modules from Corollary 7.4 and the fact that every highest weight $V_{-3 / 2}(s l(2))=H_{\theta}\left(V_{-2}\left(B_{2}\right)\right)$-module in $K L_{-3 / 2}$ is irreducible.

\section{ON THE REPRESENTATION THEORY OF $V_{2-\ell}\left(D_{\ell}\right)$}

8.1. The vertex algebra $\bar{V}_{2-\ell}\left(D_{\ell}\right)$. Let $\mathfrak{g}$ be a simple Lie algebra of type $D_{\ell}$. Recall that $2-\ell=$ $-h^{\vee} / 2+1$ is a collapsing level [4. We have the singular vector

$$
v_{n}=\left(\sum_{i=2}^{\ell} e_{\epsilon_{1}-\epsilon_{i}}(-1) e_{\epsilon_{1}+\epsilon_{i}}(-1)\right)^{n} \mathbf{1}
$$

in $V^{n-\ell+1}\left(D_{\ell}\right)$, for any $n \in \mathbb{Z}_{>0}$. As in [40], we consider the vertex algebra

$$
\bar{V}_{2-\ell}\left(D_{\ell}\right)=V^{2-\ell}\left(D_{\ell}\right) /\left\langle v_{1}\right\rangle \text {, }
$$

where $\left\langle v_{1}\right\rangle$ denotes the ideal in $V^{2-\ell}\left(D_{\ell}\right)$ generated by the singular vector $v_{1}$. We recall the following result on the classification of irreducible $\bar{V}_{2-\ell}\left(D_{\ell}\right)$-modules in the category $K L^{2-\ell}$.

Proposition 8.1. 40]

(1) The set

$$
\left\{V\left(t \omega_{\ell}\right), V\left(t \omega_{\ell-1}\right) \mid t \in \mathbb{Z}_{\geq 0}\right\}
$$

provides a complete list of irreducible finite-dimensional modules for the Zhu algebra $A\left(\bar{V}_{2-\ell}\left(D_{\ell}\right)\right)$.

(2) The set

$$
\left\{L\left((2-t-\ell) \Lambda_{0}+t \Lambda_{\ell}\right), L\left((2-t-\ell) \Lambda_{0}+t \Lambda_{\ell-1}\right) \mid t \in \mathbb{Z}_{\geq 0}\right\}
$$

provides a complete list of irreducible $\bar{V}_{2-\ell}\left(D_{\ell}\right)$-modules from the category $K L^{2-\ell}$. 
In the odd rank case $D_{2 \ell-1}$, the modules from Proposition 8.1 (2) provide a complete list of irreducible $V_{3-2 \ell}\left(D_{2 \ell-1}\right)$-modules from the category $K L_{3-2 \ell}$ (cf. 11]). The paper 11 also contains a fusion rules result in the category $K L_{3-2 \ell}$. Detailed fusion rules analysis will be presented elsewhere.

On the other hand, Theorem 4.2 implies that in the even rank case $D_{2 \ell}, V_{2-2 \ell}\left(D_{2 \ell}\right)$ is the unique irreducible $V_{2-2 \ell}\left(D_{2 \ell}\right)$-module from the category $K L_{2-2 \ell}$. In the next section we will give an explanation of this difference using singular vectors existing in the even rank case $D_{2 \ell}$.

8.2. Singular vectors in $V^{n-2 \ell+1}\left(D_{2 \ell}\right)$. In this section, we construct more singular vectors in $V^{n-2 \ell+1}\left(D_{2 \ell}\right)$. In the case $n=1$, we show that the maximal submodule of $V^{2-2 \ell}\left(D_{2 \ell}\right)$ is generated by three singular vectors. We present explicit formulas for these singular vectors.

Let $\mathfrak{g}$ be a simple Lie algebra of type $D_{2 \ell}$. Denote by $S_{2 \ell}$ the group of permutations of $2 \ell$ elements. Let

$$
\Pi_{\ell}=\left\{p \in S_{2 \ell} \mid p^{2}=1, p(i) \neq i, \forall i \in\{1, \ldots, 2 \ell\}\right\}
$$

be the set of fixed-points free involutions, which is well known to have $(2 \ell-1) ! !=1 \cdot 3 \cdot \ldots \cdot(2 \ell-1)$ elements. For $i \neq j$, denote by $(i j) \in S_{2 \ell}$ the transposition of $i$ and $j$. Then, any $p \in \Pi_{\ell}$ admits a unique decomposition of the form:

$$
p=\left(i_{1} j_{1}\right) \cdots\left(i_{\ell} j_{\ell}\right),
$$

such that $i_{h}<j_{h}$ for $1 \leq h \leq \ell$, and $i_{1}<\ldots<i_{\ell}$. Define a permutation $\bar{p} \in S_{2 \ell}$ by:

$$
\bar{p}(2 h-1)=i_{h}, \bar{p}(2 h)=j_{h}, 1 \leq h \leq \ell .
$$

Thus, we have a well defined map $p \mapsto \bar{p}$ from $\Pi_{\ell}$ to $S_{2 \ell}$. Define the function $s: \Pi_{\ell} \rightarrow\{ \pm 1\}$ as follows:

$$
s(p)=\operatorname{sign}(\bar{p}),
$$

where $\operatorname{sign}(q)$ denotes the sign of the permutation $q \in S_{2 \ell}$.

We have:

Theorem 8.2. The vector

$$
w_{n}=\left(\sum_{p \in \Pi_{\ell}} s(p) \prod_{\substack{i \in\{1, \ldots, 2 \ell\} \\ i<p(i)}} e_{\epsilon_{i}+\epsilon_{p(i)}}(-1)\right)^{n} \mathbf{1}
$$

is a singular vector in $V^{n-2 \ell+1}\left(D_{2 \ell}\right)$, for any $n \in \mathbb{Z}_{>0}$.

Proof. Direct verification of relations $e_{\epsilon_{k}-\epsilon_{k+1}}(0) w_{n}=0$, for $k=1, \ldots, 2 \ell-1, e_{\epsilon_{2 \ell-1}+\epsilon_{2 \ell}}(0) w_{n}=0$ and $e_{-\left(\epsilon_{1}+\epsilon_{2}\right)}(1) w_{n}=0$.

Remark 8.3. The vector $w_{n}$ has conformal weight $n \ell$ and its $\mathfrak{g}$-highest weight equals $2 n \omega_{2 \ell}=n\left(\epsilon_{1}+\right.$ $\left.\ldots+\epsilon_{2 \ell}\right)$. In particular, for $n=1$, the vector $w_{1}$ has conformal weight $\ell$ and highest weight $2 \omega_{2 \ell}=$ $\epsilon_{1}+\ldots+\epsilon_{2 \ell}$.

Example 8.4. Set $n=1$ for simplicity. For $\ell=2$ we recover the singular vector

$$
w_{1}=\left(e_{\epsilon_{1}+\epsilon_{2}}(-1) e_{\epsilon_{3}+\epsilon_{4}}(-1)-e_{\epsilon_{1}+\epsilon_{3}}(-1) e_{\epsilon_{2}+\epsilon_{4}}(-1)+e_{\epsilon_{1}+\epsilon_{4}}(-1) e_{\epsilon_{2}+\epsilon_{3}}(-1)\right) \mathbf{1}
$$

in $V^{-2}\left(D_{4}\right)$ of conformal weight 2 from [40. For $\ell=3$, the formula for the singular vector in $V^{-4}\left(D_{6}\right)$ of conformal weight 3 is more complicated. It is a sum of $5 ! !=15$ monomials:

$$
\begin{aligned}
& w_{1}=\left(e_{\epsilon_{1}+\epsilon_{2}}(-1) e_{\epsilon_{3}+\epsilon_{4}}(-1) e_{\epsilon_{5}+\epsilon_{6}}(-1)-e_{\epsilon_{1}+\epsilon_{2}}(-1) e_{\epsilon_{3}+\epsilon_{5}}(-1) e_{\epsilon_{4}+\epsilon_{6}}(-1)\right. \\
& +e_{\epsilon_{1}+\epsilon_{2}}(-1) e_{\epsilon_{3}+\epsilon_{6}}(-1) e_{\epsilon_{4}+\epsilon_{5}}(-1)-e_{\epsilon_{1}+\epsilon_{3}}(-1) e_{\epsilon_{2}+\epsilon_{4}}(-1) e_{\epsilon_{5}+\epsilon_{6}}(-1) \\
& +e_{\epsilon_{1}+\epsilon_{3}}(-1) e_{\epsilon_{2}+\epsilon_{5}}(-1) e_{\epsilon_{4}+\epsilon_{6}}(-1)-e_{\epsilon_{1}+\epsilon_{3}}(-1) e_{\epsilon_{2}+\epsilon_{6}}(-1) e_{\epsilon_{4}+\epsilon_{5}}(-1) \\
& +e_{\epsilon_{1}+\epsilon_{4}}(-1) e_{\epsilon_{2}+\epsilon_{3}}(-1) e_{\epsilon_{5}+\epsilon_{6}}(-1)-e_{\epsilon_{1}+\epsilon_{4}}(-1) e_{\epsilon_{2}+\epsilon_{5}}(-1) e_{\epsilon_{3}+\epsilon_{6}}(-1) \\
& +e_{\epsilon_{1}+\epsilon_{4}}(-1) e_{\epsilon_{2}+\epsilon_{6}}(-1) e_{\epsilon_{3}+\epsilon_{5}}(-1)-e_{\epsilon_{1}+\epsilon_{5}}(-1) e_{\epsilon_{2}+\epsilon_{3}}(-1) e_{\epsilon_{4}+\epsilon_{6}}(-1) \\
& +e_{\epsilon_{1}+\epsilon_{5}}(-1) e_{\epsilon_{2}+\epsilon_{4}}(-1) e_{\epsilon_{3}+\epsilon_{6}}(-1)-e_{\epsilon_{1}+\epsilon_{5}}(-1) e_{\epsilon_{2}+\epsilon_{6}}(-1) e_{\epsilon_{3}+\epsilon_{4}}(-1) \\
& +e_{\epsilon_{1}+\epsilon_{6}}(-1) e_{\epsilon_{2}+\epsilon_{3}}(-1) e_{\epsilon_{4}+\epsilon_{5}}(-1)-e_{\epsilon_{1}+\epsilon_{6}}(-1) e_{\epsilon_{2}+\epsilon_{4}}(-1) e_{\epsilon_{3}+\epsilon_{5}}(-1) \\
& \left.+e_{\epsilon_{1}+\epsilon_{6}}(-1) e_{\epsilon_{2}+\epsilon_{5}}(-1) e_{\epsilon_{3}+\epsilon_{4}}(-1)\right) \mathbf{1}
\end{aligned}
$$


Denote by $\vartheta$ the automorphism of $V^{n-2 \ell+1}\left(D_{2 \ell}\right)$ induced by the automorphism of the Dynkin diagram of $D_{2 \ell}$ of order two such that

$$
\begin{aligned}
& \vartheta\left(\epsilon_{k}-\epsilon_{k+1}\right)=\epsilon_{k}-\epsilon_{k+1}, k=1, \ldots, 2 \ell-2, \\
& \vartheta\left(\epsilon_{2 \ell-1}-\epsilon_{2 \ell}\right)=\epsilon_{2 \ell-1}+\epsilon_{2 \ell}, \vartheta\left(\epsilon_{2 \ell-1}+\epsilon_{2 \ell}\right)=\epsilon_{2 \ell-1}-\epsilon_{2 \ell} .
\end{aligned}
$$

Theorem 8.2 now implies that $\vartheta\left(w_{n}\right)$ is a singular vector in $V^{n-2 \ell+1}\left(D_{2 \ell}\right)$, for any $n \in \mathbb{Z}_{>0}$, also. The vector $\vartheta\left(w_{n}\right)$ has conformal weight $n \ell$ and its highest weight for $\mathfrak{g}$ is $2 n \omega_{2 \ell-1}=n\left(\epsilon_{1}+\ldots+\right.$ $\left.\epsilon_{2 \ell-1}-\epsilon_{2 \ell}\right)$.

We consider the associated quotient vertex algebra

$$
\widetilde{V}_{n-2 \ell+1}\left(D_{2 \ell}\right):=V^{n-2 \ell+1}\left(D_{2 \ell}\right) /\left\langle v_{n}, w_{n}, \vartheta\left(w_{n}\right)\right\rangle,
$$

where $v_{n}$ is given by relation (8.1) (for $D_{2 \ell}$ ):

$$
v_{n}=\left(\sum_{i=2}^{2 \ell} e_{\epsilon_{1}-\epsilon_{i}}(-1) e_{\epsilon_{1}+\epsilon_{i}}(-1)\right)^{n} \mathbf{1} .
$$

In particular, for $n=1$ we have the vertex algebra

$$
\widetilde{V}_{2-2 \ell}\left(D_{2 \ell}\right)=V^{2-2 \ell}\left(D_{2 \ell}\right) /\left\langle v_{1}, w_{1}, \vartheta\left(w_{1}\right)\right\rangle .
$$

Clearly, $\widetilde{V}_{2-2 \ell}\left(D_{2 \ell}\right)$ is a quotient of vertex algebra $\bar{V}_{2-2 \ell}\left(D_{2 \ell}\right)$ from Subsection 8.1. The associated Zhu algebra is

$$
A\left(\widetilde{V}_{2-2 \ell}\left(D_{2 \ell}\right)\right)=U(\mathfrak{g}) /\langle\bar{v}, \bar{w}, \vartheta(\bar{w})\rangle
$$

where

$$
\bar{v}=\sum_{i=2}^{2 \ell} e_{\epsilon_{1}-\epsilon_{i}} e_{\epsilon_{1}+\epsilon_{i}}, \quad \bar{w}=\sum_{p \in \Pi_{\ell}} s(p) \prod_{\substack{i \in\{1, \ldots, 2 \ell\} \\ i<p(i)}} e_{\epsilon_{i}+\epsilon_{p(i)}} .
$$

Lemma 8.5. We have:

(1) $\bar{w} V\left(t \omega_{2 \ell}\right) \neq 0$, for $t \in \mathbb{Z}_{>0}$.

(2) $\vartheta(\bar{w}) V\left(t \omega_{2 \ell-1}\right) \neq 0$, for $t \in \mathbb{Z}_{>0}$.

Proof. (1) Let $t=1$. Denote by $v_{\omega_{2 \ell}}$ the highest weight vector of $V\left(\omega_{2 \ell}\right)$, and by $v_{-\omega_{2 \ell}}$ the lowest weight vector of $V\left(\omega_{2 \ell}\right)$. One can easily check, using the spinor realization of $V\left(\omega_{2 \ell}\right)$, that there exists a constant $C \neq 0$ such that

$$
\bar{w}\left(v_{-\omega_{2 \ell}}\right)=C v_{\omega_{2 \ell}} .
$$

For general $t \in \mathbb{Z}_{>0}$, the claim follows using the embedding of $V\left(t \omega_{2 \ell}\right)$ into $V\left(\omega_{2 \ell}\right)^{\otimes t}$. Claim (2) follows similarly.

Theorem 8.6. We have:

(i) The trivial module $\mathbb{C}$ is the unique finite-dimensional irreducible module for $A\left(\widetilde{V}_{2-2 \ell}\left(D_{2 \ell}\right)\right)$.

(ii) $V_{2-2 \ell}\left(D_{2 \ell}\right)$ is the unique irreducible $\mathfrak{g}$-locally finite module for $\widetilde{V}_{2-2 \ell}\left(D_{2 \ell}\right)$.

(iii) The vertex operator algebra $\widetilde{V}_{2-2 \ell}\left(D_{2 \ell}\right)$ is simple, i.e.

$$
V_{2-2 \ell}\left(D_{2 \ell}\right)=V^{2-2 \ell}\left(D_{2 \ell}\right) /\left\langle v_{1}, w_{1}, \vartheta\left(w_{1}\right)\right\rangle .
$$

Proof. (i) Proposition 8.1 implies that the set

$$
\left\{V\left(t \omega_{2 \ell}\right), V\left(t \omega_{2 \ell-1}\right) \mid t \in \mathbb{Z}_{\geq 0}\right\}
$$

provides a complete list of finite-dimensional irreducible modules for the algebra $U(\mathfrak{g}) /\langle\bar{v}\rangle=A\left(\bar{V}_{2-2 \ell}\left(D_{2 \ell}\right)\right)$. Lemma 8.5 shows that $V\left(t \omega_{2 \ell}\right)$ and $V\left(t \omega_{2 \ell-1}\right)$ are not modules for $A\left(\widetilde{V}_{2-2 \ell}\left(D_{2 \ell}\right)\right)$, for $t \in \mathbb{Z}_{>0}$. Claim (i) follows. Claims (ii) and (iii) follow from (i) by applying Proposition 3.2 and Corollary 3.3 
Remark 8.7. A general character formula for certain simple affine vertex algebras at negative integer levels has been recently presented by V. G. Kac and M. Wakimoto in 37, (more precisely, $\mathfrak{g}=A_{n}, C_{n}$ for $k=-1$ and $\mathfrak{g}=D_{4}, E_{6}, E_{7}, E_{8}$ for $\left.k=-2,-3,-4,6\right)$. Note that conditions (i)-(iii) of [37. Theorem 3.1] hold for vertex algebras $V_{-b}\left(D_{n}\right), n>4, b=1, \ldots, n-2$, too. We conjecture that condition (iv) of this theorem holds as well; therefore formula (3.1) in 37 gives the character formula.

\section{Conformal embedding of $\widetilde{V}\left(-4, D_{6} \times A_{1}\right)$ into $V_{-4}\left(E_{7}\right)$}

In this section, we apply the results on representation theory of $V_{-4}\left(D_{6}\right)$ from previous sections to the conformal embedding of $\widetilde{V}\left(-4, D_{6} \times A_{1}\right)$ into $V_{-4}\left(E_{7}\right)$. This gives us an interesting example of a maximal semisimple equal rank subalgebra such that the associated conformally embedded subalgebra is not simple.

We use the construction of the root system of type $E_{7}$ from [19, 29], and the notation for root vectors similar to the notation for root vectors for $E_{6}$ from $[9]$.

For a subset $S=\left\{i_{1}, \ldots, i_{k}\right\} \subseteq\{1,2,3,4,5,6\}, i_{1}<\ldots<i_{k}$, with odd number of elements (so that $k=1,3$ or 5$)$, denote by $e_{\left(i_{1} \ldots i_{k}\right)}$ a suitably chosen root vector associated to the positive root

$$
\frac{1}{2}\left(\epsilon_{8}-\epsilon_{7}+\sum_{i=1}^{6}(-1)^{p(i)} \epsilon_{i}\right),
$$

such that $p(i)=0$ for $i \in S$ and $p(i)=1$ for $i \notin S$. We will use the symbol $f_{\left(i_{1} \ldots i_{k}\right)}$ for the root vector associated to corresponding negative root.

Note now that the subalgebra of $E_{7}$ generated by positive root vectors

$$
e_{\epsilon_{6}+\epsilon_{5}}, e_{\alpha_{1}}=e_{(1)}, e_{\alpha_{3}}=e_{\epsilon_{2}-\epsilon_{1}}, e_{\alpha_{4}}=e_{\epsilon_{3}-\epsilon_{2}}, e_{\alpha_{2}}=e_{\epsilon_{1}+\epsilon_{2}}, e_{\alpha_{5}}=e_{\epsilon_{4}-\epsilon_{3}}
$$

and the associated negative root vectors is a simple Lie algebra of type $D_{6}$. There are 30 root vectors associated to positive roots for $D_{6}$ :

$$
\begin{aligned}
& e_{\epsilon_{6}+\epsilon_{5}}, e_{\epsilon_{8}-\epsilon_{7}}, \\
& e_{(i)}, i \in\{1,2,3,4\}, \\
& e_{(i j k)}, i, j, k \in\{1,2,3,4\}, i<j<k, \\
& e_{(i 56)}, i \in\{1,2,3,4\}, \\
& e_{(i j k 56)}, i, j, k \in\{1,2,3,4\}, i<j<k, \\
& e_{ \pm \epsilon_{i}+\epsilon_{j}}, i, j \in\{1,2,3,4\}, i<j .
\end{aligned}
$$

Furthermore, the subalgebra of $E_{7}$ generated by $e_{\epsilon_{6}-\epsilon_{5}}$ and the associated negative root vector is a simple Lie algebra of type $A_{1}$. Thus, $D_{6} \oplus A_{1}$ is a semisimple subalgebra of $E_{7}$.

It follows from [3], 9] that the affine vertex algebra $\widetilde{V}\left(-4, D_{6} \times A_{1}\right)$ is conformally embedded in $V_{-4}\left(E_{7}\right)$. Remark that $\widetilde{V}\left(-4, A_{1}\right)=V_{-4}\left(A_{1}\right)$ (since $V^{-4}\left(A_{1}\right)=V_{-4}\left(A_{1}\right)$ ). This implies that $\widetilde{V}\left(-4, D_{6} \times A_{1}\right) \cong \widetilde{V}\left(-4, D_{6}\right) \otimes V_{-4}\left(A_{1}\right)$.

It was shown in 15 that

$$
\begin{aligned}
& v_{E_{7}}=\left(e_{\epsilon_{8}-\epsilon_{7}}(-1) e_{\epsilon_{6}+\epsilon_{5}}(-1)+e_{(156)}(-1) e_{(23456)}(-1)+\right. \\
& +e_{(256)}(-1) e_{(13456)}(-1)+e_{(356)}(-1) e_{(12456)}(-1)+ \\
& \left.+e_{(456)}(-1) e_{(12356)}(-1)\right) \mathbf{1}
\end{aligned}
$$

is a singular vector in $V^{-4}\left(E_{7}\right)$. Moreover,

$$
V_{-4}\left(E_{7}\right) \cong V^{-4}\left(E_{7}\right) /\left\langle v_{E_{7}}\right\rangle .
$$

Vectors $\left(e_{(12346)}(-1)\right)^{s} \mathbf{1}$, for $s \in \mathbb{Z}_{>0}$ are (non-trivial) singular vectors for the affinization of $D_{6} \oplus A_{1}$ in $V_{-4}\left(E_{7}\right)$ of highest weights $-(s+4) \Lambda_{0}+s \Lambda_{6}$ for $D_{6}^{(1)}$ and $-(s+4) \Lambda_{0}+s \Lambda_{1}$ for $A_{1}^{(1)}$. Thus there exist highest weight modules $\widetilde{L}_{D_{6}}\left(-(s+4) \Lambda_{0}+s \Lambda_{6}\right)$ and $\widetilde{L}_{A_{1}}\left(-(s+4) \Lambda_{0}+s \Lambda_{1}\right)$, for $D_{6}^{(1)}$ and $A_{1}^{(1)}$, 
respectively such that $\left(\widetilde{V}\left(-4, D_{6}\right) \otimes V_{-4}\left(A_{1}\right)\right) \cdot\left(e_{(12346)}(-1)\right)^{s} \mathbf{1}$ is isomorphic to $\widetilde{L}_{D_{6}}\left(-(s+4) \Lambda_{0}+\right.$ $\left.s \Lambda_{6}\right) \otimes \widetilde{L}_{A_{1}}\left(-(s+4) \Lambda_{0}+s \Lambda_{1}\right)$. This implies that

$$
L_{D_{6}}\left(-(s+4) \Lambda_{0}+s \Lambda_{6}\right) \otimes L_{A_{1}}\left(-(s+4) \Lambda_{0}+s \Lambda_{1}\right)
$$

are irreducible $\tilde{V}\left(-4, D_{6} \times A_{1}\right)$-modules, for $s \in \mathbb{Z}_{>0}$.

In particular, $L_{D_{6}}\left(-(s+4) \Lambda_{0}+s \Lambda_{6}\right)$ are irreducible $\left(D_{6}\right.$-locally finite) $\widetilde{V}\left(-4, D_{6}\right)$-modules, for $s \in \mathbb{Z}_{>0}$. In the next proposition, we use the notation from (8.2), 8.3), 8.4), (8.5).

Proposition 9.1. We have:

(1) Assume that $\widetilde{L}_{D_{6}}\left(-6 \Lambda_{0}+2 \Lambda_{6}\right)$ and $\widetilde{L}_{D_{6}}\left(-6 \Lambda_{0}+2 \Lambda_{5}\right)$ are highest weight $\bar{V}_{-4}\left(D_{6}\right)$-modules from the category $\mathrm{KL}^{-4}$, not necessarily irreducible. Then

$$
\widetilde{L}_{D_{6}}\left(-6 \Lambda_{0}+2 \Lambda_{6}\right) \otimes \widetilde{L}_{D_{6}}\left(-6 \Lambda_{0}+2 \Lambda_{5}\right)=0,
$$

where $\nabla$ is the tensor functor for $K L^{-4}$-modules. In other words, we cannot have a non-zero $\bar{V}_{-4}\left(D_{6}\right)$-module $M$ from $K^{-4}$ and a non-zero intertwining operator of type

$$
\left(\widetilde{L}_{D_{6}}\left(-6 \Lambda_{0}+2 \Lambda_{6}\right) \quad \widetilde{L}_{D_{6}}\left(-6 \Lambda_{0}+2 \Lambda_{5}\right)\right) .
$$

(2) Relations $w_{1} \neq 0$ and $\vartheta\left(w_{1}\right)=0$ hold in $V_{-4}\left(E_{7}\right)$. In particular, $\widetilde{V}\left(-4, D_{6}\right)$ is not simple.

Proof. For the proof of assertion (1) we first notice that the following decomposition of $D_{6}$-modules holds:

$$
\begin{aligned}
V_{D_{6}}\left(2 \omega_{6}\right) \otimes V_{D_{6}}\left(2 \omega_{5}\right)= & V_{D_{6}}\left(2 \omega_{5}+2 \omega_{6}\right) \oplus V_{D_{6}}\left(\omega_{3}+\omega_{5}+\omega_{6}\right) \oplus V_{D_{6}}\left(2 \omega_{3}\right) \\
& \oplus V_{D_{6}}\left(\omega_{1}+\omega_{5}+\omega_{6}\right) \oplus V_{D_{6}}\left(\omega_{1}+\omega_{3}\right) \oplus V_{D_{6}}\left(2 \omega_{1}\right) .
\end{aligned}
$$

Assume that $M$ is a non-zero $\bar{V}_{-4}\left(D_{6}\right)$-module in the category $K L^{-4}$ such that there is a non-trivial intertwining operator of type (9.4). Then the Frenkel-Zhu formula for fusion rules implies that $M$ must contain a non-trivial subquotient whose lowest graded component appears in the decomposition of $V_{D_{6}}\left(2 \omega_{6}\right) \otimes V_{D_{6}}\left(2 \omega_{5}\right)$. But by Proposition 8.1, the $D_{6}$-modules appearing in (9.5) cannot be lowest components of any $\bar{V}_{-4}\left(D_{6}\right)$-module. This proves assertion (1).

Assertion (1) implies that if $w_{1} \neq 0$ and $\vartheta\left(w_{1}\right) \neq 0$ in $V_{-4}\left(E_{7}\right)$, then

$$
Y\left(w_{1}, z\right) \vartheta\left(w_{1}\right)=0
$$

a contradiction since $V_{-4}\left(E_{7}\right)$ is a simple vertex algebra. The same fusion rules argument shows that if $\vartheta\left(w_{1}\right) \neq 0$ in $V_{-4}\left(E_{7}\right)$, then

$$
Y\left(\vartheta\left(w_{1}\right), z\right) e_{(12346)}(-1)^{2} \mathbf{1}=0,
$$

which again contradicts the simplicity of $V_{-4}\left(E_{7}\right)$. So, $\vartheta\left(w_{1}\right)=0$.

But if $w_{1}=0$, then, by Theorem 8.6 (iii), we have that $\widetilde{V}\left(-4, D_{6}\right)=V_{-4}\left(D_{6}\right)$. Theorem 4.2 implies that $\widetilde{V}\left(-4, D_{6}\right)$ is not simple, since the simple vertex operator algebra $V_{-4}\left(D_{6}\right)$ has only one irreducible $D_{6}$-locally finite module, a contradiction. So $w_{1} \neq 0$ and claim (2) follows.

Set

$$
\mathcal{V}_{-4}\left(D_{6}\right)=\frac{V^{-4}\left(D_{6}\right)}{<v_{1}, \vartheta\left(w_{1}\right)>}
$$

Theorem 9.2. We have:

(1) $\widetilde{V}\left(-4, D_{6}\right) \cong \mathcal{V}_{-4}\left(D_{6}\right)$.

(2) The set $\left\{L_{D_{6}}\left(-(s+4) \Lambda_{0}+s \Lambda_{6}\right) \mid \quad s \in \mathbb{Z}_{\geq 0}\right\}$ provides a complete list of irreducible $\mathcal{V}_{-4}\left(D_{6}\right)$ modules.

Proof. We first notice that $\widetilde{V}\left(-4, D_{6}\right)$ is a certain quotient of $\frac{V^{-4}\left(D_{6}\right)}{\left\langle v_{1}, \vartheta\left(w_{1}\right)\right\rangle}$, and that

$$
H_{\theta}\left(\frac{V^{-4}\left(D_{6}\right)}{<v_{1}, \vartheta\left(w_{1}\right)>}\right)=\mathcal{V}_{-2}\left(D_{4}\right) \text {. }
$$


Since $\mathcal{V}_{-2}\left(D_{4}\right)$ contains a unique non-trivial ideal which is maximal and simple, we conclude that $\frac{V^{-4}\left(D_{6}\right)}{<v_{1}, \vartheta\left(w_{1}\right)>}$ also contains a unique ideal, and it must be the ideal generated by $w_{1}$. Since in $\widetilde{V}\left(-4, D_{6}\right)$ we have that $w_{1} \neq 0$, we conclude that

$$
\widetilde{V}\left(-4, D_{6}\right) \cong \frac{V^{-4}\left(D_{6}\right)}{<v_{1}, \vartheta\left(w_{1}\right)>} .
$$

The proof of assertion (2) follows from (1), the classification result of $\bar{V}_{-4}\left(D_{6}\right)-$ modules from Proposition 8.1 and Lemma 8.5 .

\section{FUNDING}

This work was supported by the Croatian Science Foundation [grant number 2634 to D.A. and O.P.]; and the QuantiXLie Centre of Excellence, a project cofinanced by the Croatian Government and European Union through the European Regional Development Fund - the Competitiveness and Cohesion Operational Programme [KK.01.1.1.01 to D.A. and O.P.].

\section{REFERENCES}

[1] Adamović, D. "Some rational vertex algebras." Glas. Mat. Ser. III 29 (1994): 25-40.

[2] Adamović, D. "A construction of some ideals in affine vertex algebras." Int. J. Math. Math. Sci. (2003): 971-980.

[3] Adamović D., Kac V. G., Möseneder Frajria P., Papi P., and Perše O. "Finite vs infinite decompositions in conformal embeddings." Comm. Math. Phys. 348 (2016): 445-473.

[4] Adamović D., Kac V. G., Möseneder Frajria P., Papi P., and Perše O. "Conformal embeddings of affine vertex algebras in minimal $W$-algebras I: Structural results." J. Algebra 500 (2018): 117-152.

[5] Adamović D., Kac V. G., Möseneder Frajria P., Papi P., and Perše O. "Conformal embeddings of affine vertex algebras in minimal $W$-algebras II: decompositions." Jpn. J. Math. 12 (2017): 261-315.

[6] Adamović D., Kac V. G., Möseneder Frajria P., Papi P., and Perše O. "On classification of non-equal rank affine conformal embeddings and applications." Selecta Math. (N.S.) 24 (2018): 2455-2498.

[7] Adamović D., Kac V. G., Möseneder Frajria P., Papi P., and Perše O., Conformal embeddings in affine vertex superalgebras, to appear.

[8] Adamović D., and Milas A. "Vertex operator algebras associated to modular invariant representations for $A_{1}^{(1)}$." Math. Res. Lett. 2 (1995): 563-575.

[9] Adamović D., and Perše O. "Some general results on conformal embeddings of affine vertex operator algebras." Algebr. Represent. Theory 16 (2013): 51-64.

[10] Adamović D., and Perše O. "Representations of certain non-rational vertex operator algebras of affine type." $J$. Algebra 319 (2008): 2434-2450.

[11] Adamović D., and Perše O. "Fusion rules and complete reducibility of certain modules for affine Lie algebras." $J$. Algebra Appl. 13 (2014): 1350062, 18 pp.

[12] Arakawa T. "Representation theory of superconformal algebras and the Kac-Roan-Wakimoto conjecture." Duke Math. J. 130 (2005): 435-478.

[13] Arakawa T. "Associated varieties of modules over Kac-Moody algebras and $C_{2}$-cofiniteness of W-algebras." Int. Math. Res. Not. IMRN (2015): 11605-11666.

[14] Arakawa T., and Kawasetsu K. "Quasi-lisse vertex algebras and modular linear differential equations." to appear in Kostant Memorial Volume, Progress in Math. 326, Birkhauser 2018., arXiv:1610.05865.

[15] Arakawa T., and Moreau A. "Joseph ideals and lisse minimal W-algebras." J. Inst. Math. Jussieu 17 (2018): 397-417.

[16] Arakawa T., and Moreau A. "Sheets and associated varieties of affine vertex algebras." Adv. Math. 320 (2017): $157-209$.

[17] Arakawa T., and Moreau A. "On the irreducibility of associated varieties of W-algebras." J. Algebra 500 (2018): $542-568$.

[18] Borcherds R. E. "Vertex algebras, Kac-Moody algebras, and the Monster." Proc. Nat. Acad. Sci. U.S.A. 83 (1986): 3068-3071.

[19] Bourbaki. Groupes et algèbras de Lie, Paris: Hermann, 1975.

[20] Deodhar V. V., Gabber O., and Kac V. G. "Structures of some categories of representations of infinite-dimensional Lie algebras." Adv. Math. 45 (1982): 92-116.

[21] Feigin B., and Frenkel E. "Quantization of the Drinfel'd-Sokolov reduction." Phys. Lett. B 246 (1990): 75-81.

[22] Feingold A. J., and Frenkel I. B. "Classical affine algebras." Adv. Math. 56 (1985): 117-172.

[23] Frenkel E., Kac V. G., and Wakimoto M. "Characters and fusion rules for W-algebras via quantized Drinfel'dSokolov reduction." Comm. Math. Phys. 147 (1992): 295-328. 
[24] Frenkel I., Huang Y. Z., and Lepowsky J. "On axiomatic approaches to vertex operator algebras and modules." Mem. Amer. Math. Soc. 104 (1993): viii+64 pp.

[25] Frenkel I., Lepowsky J., and Meurman A. Vertex Operator Algebras and the Monster, Pure and Appl. Math. 134, Boston: Academic Press, 1988.

[26] Frenkel I., and Zhu Y.-C. "Vertex operator algebras associated to representations of affine and Virasoro algebras" Duke Math. J. 66 (1992): 123-168.

[27] Gaiotto D. "Twisted compactifications of 3d N = 4 theories and conformal blocks." arXiv:1611.01528

[28] Gorelik M., and Kac V. G. "On complete reducibility for infinite-dimensional Lie algebras." Adv. Math. 226 (2011): 1911-1972.

[29] Humphreys J. E. Introduction to Lie algebras and representation theory, Graduate Texts in Mathematics 9, New York-Berlin: Springer-Verlag, 1972.

[30] Kac V. G. "Representations of classical Lie superalgebras." in Differential geometrical methods in Mathematical Physics, II (Proc. Conf. Univ. Bonn, Bonn 1977), Springer, Berlin, 1978, 597-626.

[31] Kac V. G. Infinite dimensional Lie algebras, 3rd ed. Cambridge: Cambridge Univ. Press, 1990.

[32] Kac V. G. Vertex Algebras for Beginners, University Lecture Series, 2nd ed. AMS, Providence, RI, 1998.

[33] Kac V. G., and Peterson D. H. "Infinite-dimensional Lie algebras, theta functions and modular forms." Adv. in Math. 53 (1984): 125-264.

[34] Kac V. G., Roan S.-S., and Wakimoto M. "Quantum reduction for affine superalgebras." Comm. Math. Phys. 241 (2003): 307-342.

[35] Kac V. G., and Wakimoto M. "Quantum reduction and representation theory of superconformal algebras." Adv. Math. 185 (2004): 400-458.

[36] Kac V. G., and Wakimoto M. "On Rationality of W-algebras." Transform. Groups 13 (2008): 671-713.

[37] Kac V. G., and Wakimoto M. "On characters of irreducible highest weight modules of negative integer level over affine Lie algebras." to appear in Kostant Memorial Volume, Progress in Math. 326, Birkhauser 2018., arXiv: 1706.08387

[38] Kawasetsu K. "W-algebras with non-admissible levels and the Deligne exceptional series." Int. Math. Res. Not. $\operatorname{IMRN}(2018): 641-676$.

[39] Kazhdan D., and Lusztig G. "Tensor structures arising from affine Lie algebras, I, II." J. Amer. Math. Soc. 6 (1993): 905-947, 949-1011.

[40] Perše O. "A note on representations of some affine vertex algebras of type D." Glas. Mat. Ser. III 48 (68) (2013): 81-90.

[41] Zhu Y.-C. "Modular invariance of characters of vertex operator algebras." J. Amer. Math. Soc. 9 (1996): $237-302$. 